\title{
Vertical value-added cost information sharing in a supply chain
}

\author{
Mengqi Liu ${ }^{1}$ Yueli Zhao ${ }^{1} \cdot$ Rui Huang ${ }^{1} \cdot$ Sandun Perera ${ }^{2}$
}

Accepted: 25 February 2021

(C) The Author(s), under exclusive licence to Springer Science+Business Media, LLC, part of Springer Nature 2021

\begin{abstract}
We study a two-echelon supply chain with two homogeneous manufacturers and one common retailer who has full knowledge about his own value-added service cost structure that is unknown to the manufacturers. The retailer may choose to disclose his cost information to the manufacturers. Using a three-stage game-theoretic model, we derive optimal pricing strategies for each participant, and optimal information sharing strategies, and the optimal level of the value-added services for the retailer. Our study also reveals when the manufacturers should accept the disclosed information by the retailer. It is shown that information sharing does not always create a win-win situation among the partners in the supply chain. When the value-added service cost efficiency is low, the retailer is willing to share complete information with the manufacturers; however, information sharing harms the manufacturers' profits if they accept the shared information. In contrast, when the value-added service cost efficiency is high, the common retailer has no incentive to share information with the manufacturers and the unique equilibrium is no information sharing. Finally, we utilize a revenue-sharing contract to achieve supply chain coordination and induce information sharing under asymmetric information.
\end{abstract}

Keywords Value-added services · Information asymmetry · Game theory · Two-echelon supply chain · Equilibrium strategy

\section{Introduction}

Amid the COVID-19 pandemic, the importance of value-added services has increased as retailers compete to retain their customers who are now keen on a more convenient, safer, customized yet faster shopping experience. According to a retail service indexed commissioned by 'BookingBug' (Briggs, 2015), Apple and John Lewis are ranked as the top retailers for offering value-added services in the US and UK, respectively. These industry leaders clearly exemplify the practical importance of value-added services.

Rui Huang

huangrui_nicole@163.com

1 Business School, Hunan University, Changsha 410082, Hunan, China

2 College of Business, The University of Nevada, Reno, NV 89557, USA 
The term "Value-added" is defined as adding services or components to a product to increase its value or price (Yao et al., 2008). Specifically, value-added service means adding value to products, meeting consumers' demand, providing a competitive advantage to companies, and improving profits (Cai et al. 2019; Zhang et al., 2015). It consists of multiple services such as design, delivery, installation, training, maintenance, and financial services (Dan et al., 2018). In durable goods industries, in order to overcome the issues of product homogenization, an increasing number of companies transfer their competitive advantage to services (Armony \& Haviv, 2003; Li et al., 2014). In the home appliance industry, 'Haier Appliances' sells products through its retailers such as 'Suning' (the top home electronic appliance retailer in China) who, in turn, offers free value-added service including delivery, installation, customization, custody, and cleaning service to customers when they buy Haier's products in its stores. In the retailing industry, retailers may add value to electronic products by providing an extended warranty. For example, Wal-Mart offers an extended warranty policy after the manufacture's policy expires.

Value-added services have gained so much attention recently due to many industrial reasons. Modern businesses have shifted their focus towards more innovative models that offer products and related services simultaneously; this trend is more prominent as the profits generated by manufacturers and retailers through traditional channels has been gradually decreasing with the rapid development of e-commerce (Hartman \& Laksana, 2009). According to the 'Cifnews' reports, global e-commerce transactions broke through the $\$ 1.1$ trillion threshold in 2018. Clearly, the rapid development of e-commerce has led to the compression of traditional retail profits. As customers often evaluate products at brick-and-mortar stores to identify their "best-fit" products (Mehra et al., 2017), improving customers' satisfaction by providing value-added services has become a routine method used by suppliers and retailers. Moreover, products in many durable goods industries have become homogeneous ( $\mathrm{Li}$ et al., 2014), and firms have recognized that their competitive advantage remains not only on the price but also on the services that they provide (Armony $\&$ Haviv, 2003). Thus, firms must compete on services rather than simply lowering product prices (Lu et al., 2011). For example, household appliance buyers care about the quality and price of the products as well as the quality of the pre-sales and after-sales services. Consequently, value-added services have become one of the main product-service categories in the industry (Dai et al., 2012; Kurata \& Nam, 2010; Zhang et al., 2015).

Often, the figures on the costs associated with value-added services are considered private information of the retailer in the downstream of a supply chain. Whenever the retailer does not disclose the complete information on the cost of value-added services to its suppliers (or manufacturers), it creates an asymmetry in information shared by the partners in a supply chain. This is an interesting and understudied domain in academic and industrial research (Zhao et al., 2019; see Sect. 2 for more details). Motivated by these, we study a two-echelon supply chain that includes a common retailer (referred to as "he") and two manufacturers (referred to as "she"). The manufacturers sell their homogeneous products through the retailer who offers free value-added services along with the products to its customers. The market demand for each product is determined by its price, the price of the competitor's product as well as the level of the value-added services. Moreover, the two manufacturers do not have complete information on the retailer's cost structure regarding the value-added services. The retailer takes the decision on whether to share his cost information with the manufacturers. In this setting, we derive the optimal prices and profits for the retailer and for each manufacturer using a backward-induction approach in game theory. Moreover, we compute the optimal level for value-added services and show that this level is mainly affected by the retailer's service cost efficiency. 
We further classify retailer's cost of value-added services based on the efficiency of the process and study retailer's strategies under high and low efficiency scenarios combined with various cost information sharing settings. In this extended setting, we investigate whether

- There is an optimal service cost setup for the retailer in the market,

- There are conditions and scenarios under which the retailer has an incentive to disclose his private service cost information to upstream manufacturers,

- And when it is beneficial for the upstream manufacturers to accept the service cost information if the retailer decides to share it,

- There exists a supply chain contract that coordinates our supply chain.

In our pursuit to answer these questions, we derive the following results and insights. First, the retailer is willing to share his service cost information with both manufacturers when the service cost is inefficient, while he chooses not to share his private information with any of the manufacturers when the service cost is efficient; moreover, we show that these strategies form an equilibrium in the initial state of the game. However, interestingly, when the service cost is inefficient, none of the manufacturers are willing to accept the shared cost information. Secondly, when the cost efficiency is low, manufacturer 1 prefers no vertical information sharing, while manufacturer 2 prefers that retailer only shares information with manufacturer 1; thus, manufacturer 2 has an incentive to motivate the retailer to share the cost information with manufacturer 1 although implementing such an information-sharing mechanism through side payment may not be feasible.

The rest of this paper is organized as follows. A review of related literature is presented in the next section. Section 3 introduces our model, assumptions, and notations. In Sect. 4, we derive the optimal retail prices and the level of value-added services. The optimal wholesale prices and strategies for each manufacturer under various information sharing scenarios are discussed in Sect. 5. In Sect. 6, we compute and compare optimal profits of the retailer and the manufacturers under various scenarios and use our results to derive equilibrium strategies for the retailer in the first stage. We also numerically validate our results and show a revenue-sharing contract to coordinate our supply chain problem with asymmetric information. Section 7 concludes the paper and provides directions for future research.

\section{Literature review}

Our research model is constructed by combining two prominent features - value-added services and information asymmetry-that have been utilized in the supply chain literature. Thus, we will discuss relevant literature showcasing these two features and differentiating our work from the extant literature.

An increasing number of firms have recognized that providing service-enhanced products can gain higher profits than merely selling individual products (Xie et al., 2016). Thus, firms compete on services rather than simply lowering their prices (Li et al., 2014). Cohen and Whang (1997) establish a product life-cycle model to explore the relationship between product prices and after-sales service levels; in their model, customers can get after-sales service from either the manufacturer or an independent service shop. Yao et al., (2008) consider a supply chain consisting of one supplier and two value-added heterogeneous 
retailers, and prove the existence of equilibrium prices and added values under certain conditions. In another paper, considering the balance between a default after-sales service (offered by the manufacturer) and an optional after-sales service (offered by the retailer), Kurata and Nam (2010) analyzed whether the after-sales service plans that maximize profits produces the same service levels that satisfy customers the most. They formulate five analytical models, finding that after-sales service plans that are determined to maximize profits do not match optimal after-sales service levels that can satisfy customers the most. Dan et al. (2018) investigate a dual-channel supply chain composed of a manufacturer and a retailer, and analyze the equilibriums of the value-added service level decisions of the manufacturer and the retailer. They find that as the manufacturers' warranty service level increases, the value-added service competition is weakened, and when the warranty service level is high enough, there is no value-added service competition. They also find that the stronger the manufacturer's bargaining power, or the stronger the value-added service competition intensity, the higher the motivation for the manufacturer to provide high level warranty service. In their study, Giri and Sarker (2016) explored a supply chain system with a sole manufacturer who faces a production disruption, and two independent retailers who compete with prices and service levels; they find that a wholesale price discount scheme can coordinate the supply chain and achieve a win-win outcome. More recently, considering the influence of logistic services on consumers' channel choices, Yan et al. (2019) studied the channel structure and pricing decision of a two-echelon supply chain consisting of a manufacturer and a retailer. Zhang et al. (2019) investigate after-sale service deployment and information sharing strategies in a supply chain including a manufacturer and an independent retailer. The manufacturer decides whether it is necessary to undertake the after-sale service by herself or delegate it to the retailer, and the retailer decides whether to share his information with the manufacturer. The authors find that, compared with the situation where asymmetric information does not exist between the manufacturer and the retailer, the information advantage of the retailer may harm both parties and lead to a "prisoner's dilemma".

Despite the popularity of value-added service in practice and existing academic literature, the mechanism for sharing information about value-added service costs under asymmetric information is understudied. Our work enriches this area of research by providing guidelines on the optimal value-added service levels for retailers, the conditions under which the retailers are willing to share value-added service cost information with their suppliers, the impact of different value-added service cost information sharing mechanisms on the supply chain performance.

We now turn attention to the related literature on information asymmetry within the supply chain domain. Due to the distortion of firms' incentives and deviation of the individual decisions away from overall optimality, the cases of double marginalization, competition, and information asymmetry always lead to supply chain inefficiency (Li \& Zhang, 2008). Among them, the use of information is undoubtedly very critical in modern supply chain management. Traditionally, in a two-echelon supply chain, the retailer collects more demand information whereas the supplier collects more supply related information; however, this information is not shared among the supply chain partners, thereby creating information asymmetries and inefficiencies within the supply chain (Sahin \& Robinson, 2002). It is well known that supply chain performance is negatively affected by information asymmetry, which includes information regarding cost, demand, supply, quality, effort, risk preference, yield, capacity, etc. Shang et al. (2015) study information sharing in a supply chain with two competing manufacturers selling substitutable products through a common retailer under various scenarios with asymmetry information. They find that the retailer's 
incentive to share information strongly depends on factors such as the production cost, the intensity of competition, and the ability of the retailer to offer contracts to charge a payment for the information. Li Li et al. (2019), Li et al. (2019), Li, Chen, et al. (2019)) study the impact of information sharing on cross-sales from a contract design perspective; they consider two well-studied and widely-used contracts between manufacturers and retailers-a wholesale price contract without demand information sharing and a two-part tariff contract with demand information sharing - and find that that the two-part tariff contract can be a dominant choice under certain conditions. In a more recent study, $\mathrm{Li}, \mathrm{Wu}$, et al. (2020), $\mathrm{Li}$, Zheng, et al. (2020)) discuss the manufacturer's information acquisition and subsidy provision strategies in a supply chain consisting of two retailers with horizontal competition.

Our work is more related to the existing literature on asymmetric information about supply chain costs. Supply chain cost information asymmetries often emerge when one or more players in a supply chain have a superior level of cost-related information, such as production cost, holding cost, and ordering cost (Vosooghidizaji et al., 2020). Lau et al. (2007) examine the supply chain inefficiency caused by the information asymmetry about manufacturer's private cost in a retailer-dominated supply chain; they propose a quantity discount contract to reveal the manufacturer's private cost to coordinate the supply chain. In another paper, Yao et al. (2008) prove that the retailer's cost information should be shared between the retailer and supplier, and the supplier should make efforts to motivate the retailer to reveal his private cost information in order to achieve a 'win-win' mechanism; their supply chain includes a supplier and two heterogeneous retailers. Mukhopadhyay et al. (2008) explore the impact of cost information asymmetry in a supply chain with a wholesale price contract. They use a probability distribution to model the retailer's cost which is unknown to the manufacturer and find that information asymmetry leads to inefficiency for the manufacturer as well as for the entire supply chain. In their study, Zhao et al. (2014) find that the buyer doesn't need to share an equal amount of information with two competing suppliers as the buyer cannot achieve better performance through equal information sharing. Xie et al. (2014) analyze the value of cost information in a service supply chain when the retailer's cost type follows a probability distribution. In another study, Ma et al. (2017) evaluate the impact of cost information asymmetry in a two-echelon sustainable supply chain under a wholesale price contract and a two-part tariff contract. They derive the optimal decisions under both symmetric and asymmetric information scenarios and prove that the cost information asymmetry has larger effects under the two-part tariff contract than under the wholesale price contract. More recently, Chen and Li (2020) consider a manufacturer with private information about the cost type of production and its unobservable effort. Aiming to eliminate the negative effects on the green building market development caused by these two kinds of private information, they build a principalagent model with asymmetric information and find that the optimal subsidy of the model is obtained by introducing the 'spot check mechanism'. Lv et al. (2019) investigate an assembly system that consists of one assembler and two suppliers wherein one supplier possesses private cost information, and explores how in such a setting, the contract type (quantitypayment vs two-part tariff) and contracting sequence (simultaneous vs sequential) between the assembler and its suppliers influence the channel and individual firms' performances. In a different setting, Liu et al. (2019) explore a corporate social responsibility (CSR)-sensitive supply chain, where a contract supplier sells products through a brand retailer. The contract supplier can invest in CSR activities, but its CSR cost information is only privately known to him. Their study compares the decision effects under information asymmetry and symmetry decision, and designs a coordination mechanism that motivates the supplier to reveal the true CSR cost information. 
In contrast, we study a two-echelon supply chain with two homogeneous manufacturers and one common retailer who has his own private information on the cost associated with the value-added services; the retailer decides whether he wants to share the cost information with the manufacturers. Thus, the information asymmetry is associated with the valueadded cost and this feature (in our setting) has not been studied in the extant literature. It must be noted that the value-added cost is different from the production cost which is usually the manufacturer's private information, and manufacturers can use production cost information to optimize their own profits (Shang et al., 2015). In a model of production cost, the unit marginal cost of production is usually assumed to have a fixed value, which is generally used to find the optimal quantity of orders or inventory. Different from the models of production cost, the value-added service cost is the retailer's private information, which is always used to solve the optimal pricing problem.

\section{The model}

We study a two-echelon supply chain that involves two homogeneous manufacturers (indexed by 1 and 2) and one value-added retailer. Two manufacturers respectively sell substitutable products A and B through the retailer at different wholesale prices. It is assumed that the two manufacturers announce their wholesale prices simultaneously so that neither of them can infer information by observing the wholesale price of the competitor (Li et al., 2019; Li Li et al., 2019; Li, Chen, et al., 2019). After purchasing the products, the retailer adds value to those products and decides on the retail prices for its customers. A Stackelberg game exists between the retailer and manufacturers, and a Bertrand competition exists between the two manufacturers (Choi, 1991). The participants of the entire supply chain are risk-neutral and fully rational (Zhang \& Chen, 2013), thereby they make decisions to maximize their profits.

Each manufacturer has information on her wholesale price only, and there is no horizontal information sharing between the two competitors. Moreover, only the retailer has private information on the cost associated with the value-added services, and he can choose whether to share this cost information with the manufacturers before they announce the wholesale prices; this type of cost information sharing is referred to as the 'vertical cost information sharing' in the literature (Yao et al., 2008). Subsequently, the proposed model has asymmetric information. Moreover, we assume that there is no information leakage between different participants.

We next describe the stages of the problem and Fig. 1 below depicts these stages.

- Stage 1: The retailer decides on the services that will be added to the products and whether to share his private cost information associated with this value-added process with the manufacturers. In this stage, the retailer may decide to share his value-added service cost information with both, neither, or with either one of the two manufacturers.

- Stage 2: Whenever the cost information associated with the value-added services is shared by the retailer, each manufacturer first decides whether to accept this information. Then, the manufactures simultaneously announce their profit-maximizing wholesale prices.

- Stage 3: In response to the wholesale prices, the retailer announces the profit-maximizing retail prices. Finally, demand will be realized, and the production will be completed satisfying the demand. 


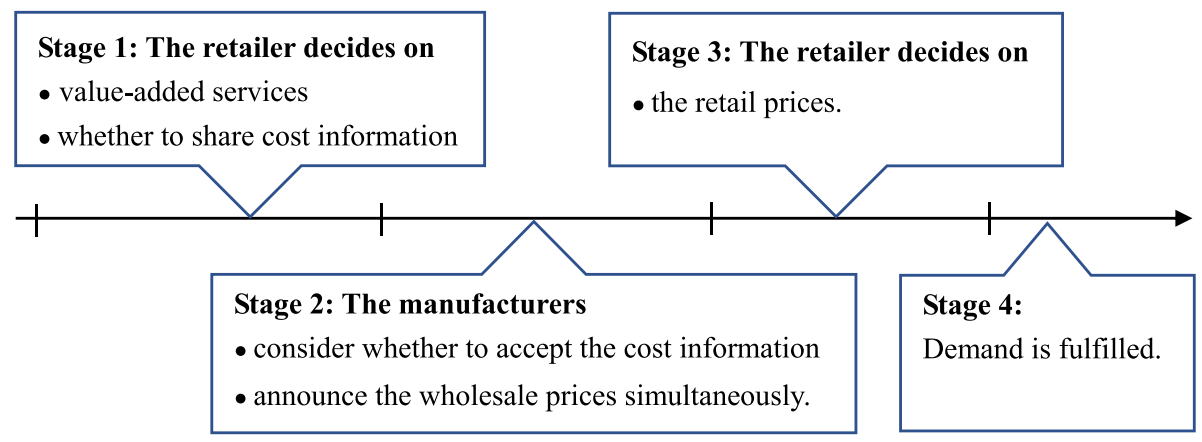

Fig. 1 Timeline of decisions and events

\subsection{Two-echelon supply chain system}

As shown in Fig. 2 below, two manufacturers sell products A and B to a common retailer at wholesale prices $\mathrm{w}_{1}$ and $\mathrm{w}_{2}$, respectively. These products are fully substitutable. The retailer provides the same level $(\nu)$ of value-added service on the products and sells them to customers at retail prices $p_{1}$ and $p_{2}$, respectively. Since the retailer utilizes the same human, material, and financial resources to provide value-added services, it is reasonable to assume the same level of value-added services on both products A and B; however, this assumption can be relaxed in a more general setting.

\subsection{Demand functions}

For convenience, we employ linear demand functions which have been widely used in supply chain literature (Li et al., 2019; Li Li et al., 2019; Li, Chen, et al., 2019; Xing et al. 2019; Yan

Fig. 2 The two-echelon supply chain system

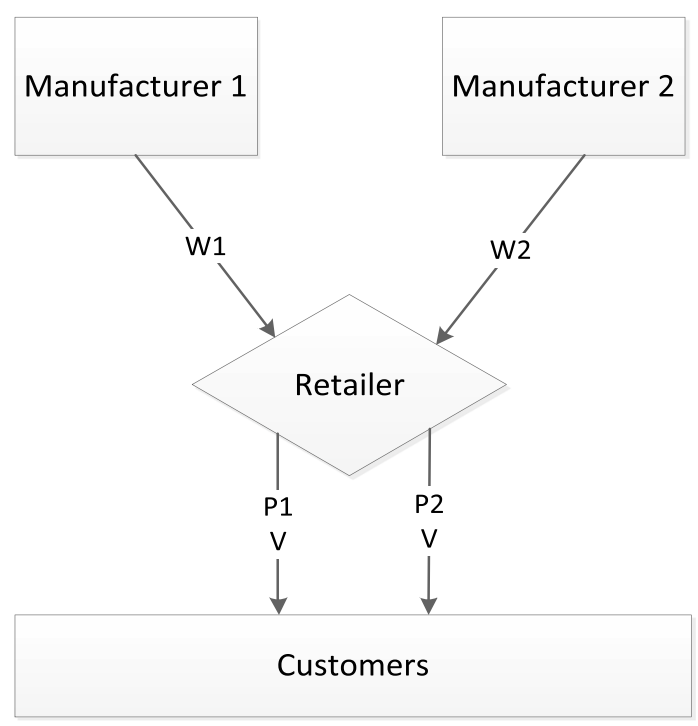


et al., 2016; Yao et al., 2008) and operations management literature (Li \& Zhang, 2008). The demand for a product depends on its price, the level of value-added services, and the competitor's price (mainly due to substitution). Assuming that the potential (basic) market demands of products A and B are sufficiently large (Yao et al., 2008), the demands of products A and B, which we denote respectively by $d_{1}$ and $d_{2}$, are calculated as:

$$
\begin{aligned}
& d_{1}=\alpha_{1}-\beta_{1} p_{1}+\beta_{2} v+k\left(p_{2}-p_{1}\right) ; \\
& d_{2}=\alpha_{2}-\beta_{1} p_{2}+\beta_{2} v+k\left(p_{1}-p_{2}\right),
\end{aligned}
$$

where $\alpha_{1}$ and $\alpha_{2}$ are the basic market demands of products $\mathrm{A}$ and $\mathrm{B}$, respectively; $\beta_{1}>0$ is the price sensitivity coefficient and $\beta_{2}>0$ is the value-added service level sensitivity coefficient of customers. Moreover, $k$ is the cross-price sensitivity of customers that emerges due to the price difference, which takes a value between 0 and $1(0<k<1)$ to ensure that the demand curve leans downward.

Table 1 below shows the notations we use in this paper.

\subsection{Profit functions}

We describe the profit functions of the manufacturers and the retailer in this subsection. Since the retailer's order quantity is equal to the market demand and the supply is instantaneous, there is no inventory cost for the manufacturers (Dan et al., 2018). We assume that the manufacturers' production capacity is infinite, which ensures that they can satisfy the entire requirement, and the unit production cost is fixed (Yao et al., 2008). The marginal production cost of the $i$-th manufacturer is denoted by $\mathrm{c}_{\mathrm{M}_{\mathrm{i}}}(\mathrm{i}=1,2)$ and assumed to be zero without loss of generality ( $\mathrm{Li}, \mathrm{Wu}$, et al., 2020; Li, Zheng, et al., 2020). Thus, the profit functions of two manufacturers can be written as

$$
\begin{aligned}
& \pi_{M_{1}}=d_{1} w_{1} ; \\
& \pi_{M_{2}}=d_{2} w_{2} .
\end{aligned}
$$

We utilize the value-added service cost function, $c_{i()}$ (for $\mathrm{i}=1,2$ ), to describe the relationship between the added value and corresponding value-added service cost. It is assumed that the retailer provides the same value-added services for both products $\mathrm{A}$ and B. Since the retailer utilizes the same resources (including human, material, and financial resources) to provide value-added services, the value-added service efficiency, $\mu_{1}$, is assumed to be the same. We adopt the following widely used service cost function (Tsay \& Agrawal, 2000; Yao et al., 2008) in our analysis:

$$
c_{i(v)}=\frac{\mu_{1}}{2} v^{2}, \quad i=1,2 .
$$

Note that this functional form of the cost function has the desirable properties below:

$$
\frac{\mathrm{d} c_{i(v)}}{d v}>0, \quad \frac{d^{2} c_{i(v)}}{d v^{2}}>0 .
$$

It must be noted that $\mu_{1}$ in Eq. (5) above is the realized value of a random variable $\mu$, where $\mu$ represents the efficiency of the retailer in the value-added process; a smaller value 
Table 1 Notations

\begin{tabular}{|c|c|}
\hline Notation & Explanation \\
\hline$\alpha_{\mathrm{i}}$ & The basic demand in the market for product $\mathrm{A}$ and $\mathrm{B}, \mathrm{i}=1,2$ \\
\hline$\beta_{1}$ & Price sensitivity coefficient $\left(\beta_{1}>0\right)$ \\
\hline$\beta_{2}$ & Value-added service level sensitivity coefficient $\left(\beta_{2}>0\right)$ \\
\hline $\mathrm{p}_{1}$ & The retail price of product $\mathrm{A}$ \\
\hline $\mathrm{p}_{2}$ & The retail price of product $B$ \\
\hline $\mathrm{k}$ & Cross price sensitivity of customers $(0<\mathrm{k}<1)$ \\
\hline$d_{i}$ & Market demand for product $\mathrm{A}$ and $\mathrm{B}, \mathrm{i}=1,2$ \\
\hline $\mathrm{w}_{\mathrm{i}}$ & The wholesale price of product $\mathrm{A}$ and $\mathrm{B}, \mathrm{i}=1,2$ \\
\hline $\mathrm{v}$ & Value-added service level \\
\hline$\pi_{M_{i}}$ & Profit of manufacturer $\mathrm{i}, \mathrm{i}=1,2$ \\
\hline$\pi_{\mathrm{R}}$ & Profit of retailer \\
\hline$\pi_{\mathrm{SC}}$ & Profit of supply chain \\
\hline $\mathrm{c}_{\mathrm{i}}(\mathrm{v})$ & Value-added service cost of product $\mathrm{A}$ and $\mathrm{B}, \mathrm{i}=1,2$ \\
\hline $\mathbf{c}_{\mathbf{M}_{\mathrm{i}}}$ & The production cost of manufacturer $\mathrm{i}, \mathrm{i}=1,2$ \\
\hline$\mu$ & The efficiency of the retailer in his value-added process; $\mu$ is a random variable \\
\hline$\mu_{1}$ & Realized value of $\mu$ which is observed privately by the retailer \\
\hline $\bar{\mu}$ & Average value-added service cost efficiency of the industry \\
\hline$\varepsilon$ & Deviation of the value-added service cost-efficiency \\
\hline$c_{s}$ & Information transmission cost of the retailer \\
\hline$\{\mathrm{S}, \mathrm{N}, \mathrm{P}\}$ & $\begin{array}{l}\text { Superscript for the scenarios with "sharing information with both manufactur- } \\
\text { ers", "sharing information with no manufacturer", and "sharing information } \\
\text { with only one manufacturer" }\end{array}$ \\
\hline$h$ & The indicator when the cost efficiency is high, i.e., $\mu \in U[\bar{\mu}-\varepsilon, \bar{\mu}]$ \\
\hline$l$ & The indicator when the cost efficiency is low, i.e., $\mu \in U[\bar{\mu}, \bar{\mu}+\varepsilon]$ \\
\hline $\mathrm{p}_{\mathrm{i}}^{*}$ & Equilibrium retail price, $\mathrm{i}=1,2$ \\
\hline $\mathrm{w}_{\mathrm{i}}^{*}$ & Equilibrium wholesale price, $\mathrm{i}=1,2$ \\
\hline$R S C$ & Superscript for the profit under "Revenue sharing contract" \\
\hline
\end{tabular}

of $\mu$ implies a more efficient retailer. In the entire supply chain, only the retailer knows the true value of $\mu$, and he decides whether to share this service cost information with the two manufacturers. Although the manufacturers do not know the true value of $\mu$, they can estimate the probability distribution of this parameter using available information. As in Yao et al. (2008), we assume $\mu$ is uniformly distributed, i.e., $\mu \in U[\bar{\mu}-\varepsilon, \bar{\mu}+\varepsilon]$; the average value-added service cost efficiency is $\bar{\mu}$ and the deviation is $\varepsilon$, and these parameters can be estimated by each participant in the supply chain. If the retailer decides to share cost information with the manufacturers, then he will specify a range of values within which $\mu$ lies, specifically, the retail will indicate $\mu \in U[\bar{\mu}-\varepsilon, \bar{\mu}]$ or $\mu \in U[\bar{\mu}, \bar{\mu}+\varepsilon]$. Since $\mu \in U[\bar{\mu}-\varepsilon, \bar{\mu}]$ and $\mu \in U[\bar{\mu}, \bar{\mu}+\varepsilon]$ respectively imply that the retailer's value-added services are more and less efficient, we denote these scenarios by high $(h)$ and low $(l)$, respectively (Zhao et al., 2014).

Information sharing and value-added services are long-term decisions. This is because if a retailer agrees to share information, then he should set up systems for information transmission. The cost $\left(c_{s}\right)$ of setting up an information transmission system is sunk cost, 
and we ignore this cost to simplify the analysis (Zhou et al., 2017). Thus, the profit function of the retailer is

$$
\pi_{R}=\left(p_{1}-w_{1}-c_{i}(v)\right) d_{1}+\left(p_{2}-w_{2}-c_{i}(v)\right) d_{2} .
$$

As mentioned earlier, this is a three-stage game, and we analyze the dynamic game with the backward induction approach. First, we assume the wholesale prices are given and find the equilibrium retail prices and added values in stage 3. Next, we find the manufacturers' optimal decision in stage 2 given the best response functions obtained in stage 3 . Finally, we turn attention to the retailer's information revelation mechanism in stage 1 .

\section{Retailer's best response}

In our backward induction approach, we first solve the retailer's problem. Since there is no horizontal information sharing between two competing manufacturers and no information leakage in the supply chain, only the retailer has full information about his service cost structure. The objective of the retailer is to find the optimal retail prices $p_{i}$ and value-added service level $\nu$ to maximize his profit given the wholesale prices, $w_{i}$ for $\mathrm{i}=1,2$. Thus, for $\mathrm{i}=1,2$, the retailer's problem is

$$
\underset{p_{i}, v}{\operatorname{Max}} E\left[\pi_{R} \mid w_{i}\right]=\underset{p_{i}, v}{\operatorname{Max}} E\left[\left(p_{1}-w_{1}-c_{i}(v)\right) d_{1}+\left(p_{2}-w_{2}-c_{i}(v)\right) d_{2} \mid w_{i}\right] .
$$

Assuming that the retailer decides the retail prices simultaneously for products A and B, we get the following result. (All proofs are given in the "Appendix".)

Lemma 1 The optimal retail prices and value-added service level for products $A$ and $B$ can be computed simultaneously using:

$$
\begin{aligned}
p_{1}^{*} & =\frac{3 \beta_{1} \beta_{2}^{2}+6 \beta_{2}^{2} k+2 \beta_{1}^{3} \mu_{1} w_{1}+2 \alpha_{1} \beta_{1}^{2} \mu_{1}+2 \alpha_{1} \beta_{1} k \mu_{1}+2 \alpha_{2} \beta_{1} k \mu_{1}+4 \beta_{1}^{2} k \mu_{1} w_{1}}{4 \beta_{1}^{2} \mu_{1}\left(\beta_{1}+2 k\right)}, \\
p_{2}^{*} & =\frac{3 \beta_{1} \beta_{2}^{2}+6 \beta_{2}^{2} k+2 \beta_{1}^{3} \mu_{1} w_{2}+2 \alpha_{2} \beta_{1}^{2} \mu_{1}+2 \alpha_{1} \beta_{1} k \mu_{1}+2 \alpha_{2} \beta_{1} k \mu_{1}+4 \beta_{1}^{2} k \mu_{1} w_{2}}{4 \beta_{1}^{2} \mu_{1}\left(\beta_{1}+2 k\right)}, \\
v & =\frac{\beta_{2}}{\beta_{1} \mu_{1}} .
\end{aligned}
$$

Thus, in the equilibrium, there exists an optimal price for each product and an optimal level for the value-added services. Moreover, the optimal value added to the products is independent of the wholesale prices and is directly affected by the retailer's own service cost efficiency $\left(\mu_{1}\right)$. In particular, our results suggest that more efficient retailers (in terms of their valueadded service cost) should add more value to the products via value-added services.

It is easily seen that $\frac{\partial p_{i}^{*}}{\partial w_{i}}=\frac{1+2 \mathrm{k}}{2\left(\beta_{1}+2 k\right)}>0$, and thus, the higher the wholesale price, the more the retailer tends to charge the customers. Although this is an intuitive observation, it is consistent with the practice.

Furthermore, note that $\frac{\partial p_{i}^{*}}{\partial \mu_{1}}=-\frac{3 \beta_{2}^{2}}{4 \beta_{1}^{2} \mu_{1}^{2}}<0$. This means that retailers with higher efficiencies (with regard to their value-added service cost) charge a higher retail price. This 
result is partly driven by the fact that these retailers also add more value to the products in the first place. One clear implication of our results is that the retailers should strive to improve service efficiency in order to achieve higher revenues.

Next, we investigate manufacturers' optimal decisions with respect to the retailer's response function under five different scenarios.

\section{Manufacturers' equilibrium strategy}

In this section, we analyze the two manufacturers' equilibrium strategies under different information sharing scenarios about the retailer's cost of value-added services. Superscripts $S, N$, and $P$ are used to represent three-complete, no, and partial- information sharing scenarios, respectively.

Based on the available information about the retailer's value-added service cost, each manufacturer $i(i=1,2)$ needs to decide the wholesale price $w_{i}$ that maximizes her profit. Recall that each manufacturer has zero marginal production cost (Lin \& Parlaktürk, 2012; Mehra et al., 2017). So, for $i=1,2$, the $\mathrm{i}$-th manufacturer's expected profit is

$$
\underset{w_{i}}{\operatorname{Max}} E\left[\pi_{M_{i}}\right]=\underset{w_{i}}{\operatorname{Max}} E\left[d_{i} w_{i}\right]
$$

Depending on the retailer's decision about sharing his private cost information with the manufacturers, there are five different scenarios:

- $\quad N$ : The retailer shares cost information with none of the manufacturers.

- Sh: The retailer shares cost information with both manufacturers and $\mu \in U[\bar{\mu}-\varepsilon, \bar{\mu}]$.

- Sl: The retailer shares cost information with both manufacturers and $\mu \in U[\bar{\mu}, \bar{\mu}+\varepsilon]$.

- Ph: The retailer shares cost information with only one manufacturer (specifically refers to manufacturer 1$)$, and $\mu \in U[\bar{\mu}-\varepsilon, \bar{\mu}]$.

- Pl: The retailer shares cost information with only one manufacturer (specifically refers to manufacturer 1$)$, and $\mu \in U[\bar{\mu}, \bar{\mu}+\varepsilon]$.

From now on, we will use the specific labels above as superscripts to denote the wholesale prices and profits under corresponding information sharing scenarios.

\subsection{No information sharing $(N)$}

Under this scenario, the retailer does not share the cost information with the manufacturers in the first stage. So, the manufacturers do not have any knowledge of the service cost information. Since there is no information sharing and information leakage between the two manufacturers, they also do not know whether their rival knows the cost information. Consequently, manufacturers make wholesale price decisions assuming that the rival has the same information, i.e., $\mu \in U[\bar{\mu}-\varepsilon, \bar{\mu}+\varepsilon]$. Hence, for $\mathrm{i}=1,2$, the $\mathrm{i}$-th manufacturer's expected profit in this case is 


$$
\underset{w_{i}}{\operatorname{Max}} E\left[\pi_{M_{i}}\right]=\operatorname{Max}_{w_{i}} \int_{\bar{\mu}-\varepsilon}^{\bar{\mu}+\varepsilon} d_{i} w_{i} d F_{\mu[\bar{\mu}-\varepsilon, \bar{\mu}+\varepsilon]}\left(\mu_{1}\right)
$$

where $d F_{\mu[\bar{\mu}-\varepsilon, \bar{\mu}+\varepsilon]}(\cdot)$ is the distribution function of $U[\bar{\mu}-\varepsilon, \bar{\mu}+\varepsilon]$. In general, we denote the distribution function of $U[a, b]$ by $d F_{\mu[a, b]}(\cdot)$ throughout this paper. The solution to the above maximization problem is presented in the following lemma.

Lemma 2 The manufacturers' wholesale prices in the equilibrium under no information sharing scenario are:

$$
\begin{aligned}
& w_{1}^{N *}=\frac{2 \beta_{1} \beta_{2}^{2} \ln \left(\frac{\bar{\mu}+\varepsilon}{\bar{\mu}-\varepsilon}\right)+3 \beta_{2}^{2} k \ln \left(\frac{\bar{\mu}+\varepsilon}{\bar{\mu}-\varepsilon}\right)+8 \alpha_{1} \beta_{1}^{2} \varepsilon+8 \alpha_{1} \beta_{1} k \varepsilon+4 \alpha_{2} \beta_{1} k \varepsilon}{4 \beta_{1} \varepsilon\left(4 \beta_{1}^{2}+8 \beta_{1} k+3 k^{2}\right)} ; \\
& w_{2}^{N *}=\frac{2 \beta_{1} \beta_{2}^{2} \ln \left(\frac{\bar{\mu}+\varepsilon}{\bar{\mu}-\varepsilon}\right)+3 \beta_{2}^{2} k \ln \left(\frac{\bar{\mu}+\varepsilon}{\bar{\mu}-\varepsilon}\right)+8 \alpha_{2} \beta_{1}^{2} \varepsilon+8 \alpha_{2} \beta_{1} k \varepsilon+4 \alpha_{1} \beta_{1} k \varepsilon}{4 \beta_{1} \varepsilon\left(4 \beta_{1}^{2}+8 \beta_{1} k+3 k^{2}\right)} .
\end{aligned}
$$

Lemma 2 provides the wholesale prices when manufacturers passively accept the information sharing decision of the retailer. Since it can be shown (see Appendix A-2) that

$$
\left[\varepsilon^{2} \ln \left(\frac{\bar{\mu}+\varepsilon}{\bar{\mu}-\varepsilon}\right)-\bar{\mu}^{2} \ln \left(\frac{\bar{\mu}+\varepsilon}{\bar{\mu}-\varepsilon}\right)+2 \varepsilon \bar{\mu}\right]>0,
$$

and $(\varepsilon-\bar{\mu})<0$, we have that

$$
\frac{\partial w_{i}^{N *}}{\partial \varepsilon}=\frac{-\beta_{2}^{2}\left(2 \beta_{1}+3 k\right)\left[\varepsilon^{2} \ln \left(\frac{\bar{\mu}+\varepsilon}{\bar{\mu}-\varepsilon}\right)-\bar{\mu}^{2} \ln \left(\frac{\bar{\mu}+\varepsilon}{\bar{\mu}-\varepsilon}\right)+2 \varepsilon \bar{\mu}\right]}{4 \beta_{1} \varepsilon^{2}\left(\varepsilon^{2}-\bar{\mu}^{2}\right)\left(4 \beta_{1}^{2}+8 \beta_{1} k+3 k^{2}\right)}>0 .
$$

Thus, when the distribution of the cost of the value-adding service is more dispersed, the manufacturers should set a higher wholesale price.

Finally, we observe that

$$
\frac{\partial w_{i}^{N *}}{\partial \bar{\mu}}=\frac{\beta_{2}^{2}}{2 \beta_{1} \varepsilon\left(\varepsilon^{2}-\bar{\mu}^{2}\right)\left(2 \beta_{1}+k\right)}<0,
$$

where the value of $\bar{\mu}$ represents the market (and manufacturers') estimate of the average value of the cost efficiency of the retailer. Moreover, as we discussed earlier, the smaller values of $\bar{\mu}$ are associated with more efficient retailers. Interestingly, $\frac{\partial w_{i}^{N *}}{\partial \bar{\mu}}<0$ implies that if manufacturers believe that the retailer's cost efficiency is low on average, then they should lower the wholesale price. It can be easily verified that this observation is common in all five scenarios- $N, S h, S l, P h$, and $P l$-and thus, it is a robust result in our setting. However, we will avoid recurrent discussion on this result under other scenarios for brevity.

Next, we will present the analysis under the remaining four- $S h, S l, P h$, and $P l-$ scenarios first and discuss their implications at the end of Sect. 5.5. 


\subsection{Sharing cost information when the efficiency is high (Sh)}

Here, the retailer shares cost information with both the manufacturers in the first stage. So, two manufacturers have full knowledge of the service cost information, that is, manufacturers know that $\mu \in U[\bar{\mu}-\varepsilon, \bar{\mu}]$. Again, the manufacturers make their wholesale pricing decisions assuming that the rival has the same information. In this case, for $i=1,2$, the $i$-th manufacturer's expected profit is

$$
\underset{w_{i}}{\operatorname{Max}} E\left[\pi_{M_{i}}\right]=\operatorname{Max}_{w_{i}} \int_{\bar{\mu}-\varepsilon}^{\bar{\mu}} d_{i} w_{i} d F_{\mu[\bar{\mu}-\varepsilon, \bar{\mu}]}\left(\mu_{1}\right)
$$

Thus, we can derive the following result.

Lemma 3 The manufacturers' wholesale prices in the equilibrium under complete information sharing, when the cost efficiency is high (Sh), are:

$$
\begin{aligned}
w_{1}^{S h *} & =\frac{2 \beta_{1} \beta_{2}^{2} \ln \left(\frac{\bar{\mu}}{\bar{\mu}-\varepsilon}\right)+3 \beta_{2}^{2} k \ln \left(\frac{\bar{\mu}}{\bar{\mu}-\varepsilon}\right)+4 \alpha_{1} \beta_{1}^{2} \varepsilon+4 \alpha_{1} \beta_{1} k \varepsilon+2 \alpha_{2} \beta_{1} k \varepsilon}{2 \beta_{1} \varepsilon\left(4 \beta_{1}^{2}+8 \beta_{1} k+3 k^{2}\right)}, \\
w_{2}^{S h *} & =\frac{2 \beta_{1} \beta_{2}^{2} \ln \left(\frac{\bar{\mu}}{\bar{\mu}-\varepsilon}\right)+3 \beta_{2}^{2} k \ln \left(\frac{\bar{\mu}}{\bar{\mu}-\varepsilon}\right)+4 \alpha_{2} \beta_{1}^{2} \varepsilon+2 \alpha_{1} \beta_{1} k \varepsilon+4 \alpha_{2} \beta_{1} k \varepsilon}{2 \beta_{1} \varepsilon\left(4 \beta_{1}^{2}+8 \beta_{1} k+3 k^{2}\right)} .
\end{aligned}
$$

\subsection{Sharing cost information when the efficiency is low (SI)}

For this scenario, the retailer shares cost information with both the manufacturers in the first stage. Thus, the manufacturers know that $\mu \in U[\bar{\mu}, \bar{\mu}+\varepsilon]$. Assuming that the information is available to both manufacturers, the maximization problem of the $i$-th $(i=1,2)$ manufacturer is

$$
\underset{w_{i}}{\operatorname{Max}} E\left[\pi_{M_{i}}\right]=\operatorname{Max}_{w_{i}} \int_{\bar{\mu}}^{\bar{\mu}+\varepsilon} d_{i} w_{i} d F_{\mu[\bar{\mu}, \bar{\mu}+\varepsilon]}\left(\mu_{1}\right),
$$

and the equilibrium prices are given in the following lemma.

Lemma 4 The manufacturers' wholesale prices in the equilibrium under complete information sharing, when the cost efficiency is low (Sl), are:

$$
\begin{aligned}
w_{1}^{S l *} & =\frac{2 \beta_{1} \beta_{2}^{2} \ln \left(\frac{\bar{\mu}+\varepsilon}{\bar{\mu}}\right)+3 \beta_{2}^{2} k \ln \left(\frac{\bar{\mu}+\varepsilon}{\bar{\mu}}\right)+4 \alpha_{1} \beta_{1}^{2} \varepsilon+4 \alpha_{1} \beta_{1} k \varepsilon+2 \alpha_{2} \beta_{1} k \varepsilon}{2 \beta_{1} \varepsilon\left(4 \beta_{1}^{2}+8 \beta_{1} k+3 k^{2}\right)}, \\
w_{2}^{S l *} & =\frac{2 \beta_{1} \beta_{2}^{2} \ln \left(\frac{\bar{\mu}+\varepsilon}{\bar{\mu}}\right)+3 \beta_{2}^{2} k \ln \left(\frac{\bar{\mu}+\varepsilon}{\bar{\mu}}\right)+4 \alpha_{2} \beta_{1}^{2} \varepsilon+2 \alpha_{1} \beta_{1} k \varepsilon+4 \alpha_{2} \beta_{1} k \varepsilon}{2 \beta_{1} \varepsilon\left(4 \beta_{1}^{2}+8 \beta_{1} k+3 k^{2}\right)} .
\end{aligned}
$$




\subsection{Partially sharing cost information when the efficiency is high (Ph)}

Under this scenario, the retailer only shares cost information, i.e., $\mu \in U[\bar{\mu}-\varepsilon, \bar{\mu}]$, with one manufacturer (manufacturer 1) in the first stage. So, manufacturer 1 makes her decisions with the full cost information whereas manufacturer 2 makes her decisions based on the market predictions, i.e., $\mu \in U[\bar{\mu}-\varepsilon, \bar{\mu}+\varepsilon]$. Nevertheless, since there is no horizontal information sharing and no information leakage between manufacturers, each manufacturer makes wholesale price decisions assuming that the rival has the same information. Consequently, the manufacturers' maximization problems are as follows:

$$
\begin{aligned}
& \underset{w_{1}}{\operatorname{Max}} E\left[\pi_{M_{1}}\right]=\operatorname{Max}_{w_{1}} \int_{\bar{\mu}-\varepsilon}^{\bar{\mu}} d_{1} w_{1} d F_{\mu[\bar{\mu}-\varepsilon, \bar{\mu}]}\left(\mu_{1}\right), \\
& \underset{w_{2}}{\operatorname{Max}} E\left[\pi_{M_{2}}\right]=\operatorname{Max}_{w_{2}} \int_{\bar{\mu}-\varepsilon}^{\bar{\mu}+\varepsilon} d_{2} w_{2} d F_{\mu[\bar{\mu}-\varepsilon, \bar{\mu}+\varepsilon]}\left(\mu_{1}\right) .
\end{aligned}
$$

Lemma 5 The manufacturers' wholesale prices in the equilibrium under partial information sharing, when the cost efficiency is high $(P h)$, are:

$$
\begin{aligned}
& w_{1}^{P h *}=\frac{4 \beta_{1} \beta_{2}^{2} \ln \left(\frac{\bar{\mu}}{\bar{\mu}-\varepsilon}\right)+4 \beta_{2}^{2} k \ln \left(\frac{\bar{\mu}}{\bar{\mu}-\varepsilon}\right)+\beta_{2}^{2} k \ln \left(\frac{\bar{\mu}+\varepsilon}{\bar{\mu}-\varepsilon}\right)+8 \alpha_{1} \beta_{1}^{2} \varepsilon+8 \alpha_{1} \beta_{1} k \varepsilon+2 \alpha_{2} \beta_{1} k \varepsilon}{4 \beta_{1} \varepsilon\left(4 \beta_{1}^{2}+8 \beta_{1} k+3 k^{2}\right)}, \\
& w_{2}^{P h *}=\frac{\beta_{1} \beta_{2}^{2} \ln \left(\frac{\bar{\mu}+\varepsilon}{\bar{\mu}-\varepsilon}\right)+\beta_{2}^{2} k \ln \left(\frac{\bar{\mu}}{\bar{\mu}-\varepsilon}\right)+\beta_{2}^{2} k \ln \left(\frac{\bar{\mu}+\varepsilon}{\bar{\mu}-\varepsilon}\right)+4 \alpha_{2} \beta_{1}^{2} \varepsilon+2 \alpha_{1} \beta_{1} k \varepsilon+4 \alpha_{2} \beta_{1} k \varepsilon}{2 \beta_{1} \varepsilon\left(4 \beta_{1}^{2}+8 \beta_{1} k+3 k^{2}\right)} .
\end{aligned}
$$

\subsection{Partially sharing cost information when the efficiency is low (PI)}

Here, in the first stage, the retailer only shares cost information, i.e., $\mu \in U[\bar{\mu}, \bar{\mu}+\varepsilon]$, with one manufacturer (manufacturer 1). Again, manufacturer 1 makes her decision with the full information whereas manufacturer 2 makes decisions based on the market predictions, i.e., $\mu \in U[\bar{\mu}-\varepsilon, \bar{\mu}+\varepsilon]$. Thus, manufacturers 1 and 2 respectively solve

$$
\begin{gathered}
\operatorname{Max}_{w_{1}} E\left[\pi_{M_{1}}\right]=\operatorname{Max}_{w_{1}} \int_{\bar{\mu}}^{\bar{\mu}+\varepsilon} d_{1} w_{1} d F_{\mu[\bar{\mu}, \bar{\mu}+\varepsilon]}\left(\mu_{1}\right), \quad \text { and } \\
\operatorname{Max}_{w_{2}} E\left[\pi_{M_{2}}\right]=\operatorname{Max}_{w_{2}}^{\bar{\mu}+\varepsilon} \int_{\bar{\mu}-\varepsilon}^{\bar{\mu}} d_{2} w_{2} d F_{\mu[\bar{\mu}-\varepsilon, \bar{\mu}+\varepsilon]}\left(\mu_{1}\right) .
\end{gathered}
$$

Moreover, the optimal wholesale prices are given in the following lemma. 
Lemma 6 The manufacturers' wholesale prices in the equilibrium under partial information sharing, when the cost efficiency is low (Pl), are:

$$
\begin{aligned}
& w_{1}^{P l *}=\frac{4 \beta_{1} \beta_{2}^{2} \ln \left(\frac{\bar{\mu}+\varepsilon}{\bar{\mu}}\right)+4 \beta_{2}^{2} k \ln \left(\frac{\bar{\mu}+\varepsilon}{\bar{\mu}}\right)+\beta_{2}^{2} k \ln \left(\frac{\bar{\mu}+\varepsilon}{\bar{\mu}-\varepsilon}\right)+8 \alpha_{1} \beta_{1}^{2} \varepsilon+8 \alpha_{1} \beta_{1} k \varepsilon+4 \alpha_{2} \beta_{1} k \varepsilon}{4 \beta_{1} \varepsilon\left(4 \beta_{1}^{2}+8 \beta_{1} k+3 k^{2}\right)}, \\
& w_{2}^{P l *}=\frac{\beta_{1} \beta_{2}^{2} \ln \left(\frac{\bar{\mu}+\varepsilon}{\bar{\mu}-\varepsilon}\right)+\beta_{2}^{2} k \ln \left(\frac{\bar{\mu}+\varepsilon}{\bar{\mu}}\right)+\beta_{2}^{2} k \ln \left(\frac{\bar{\mu}+\varepsilon}{\bar{\mu}-\varepsilon}\right)+4 \alpha_{2} \beta_{1}^{2} \varepsilon+2 \alpha_{1} \beta_{1} k \varepsilon+4 \alpha_{2} \beta_{1} k \varepsilon}{2 \beta_{1} \varepsilon\left(4 \beta_{1}^{2}+8 \beta_{1} k+3 k^{2}\right)} .
\end{aligned}
$$

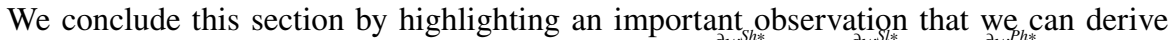
$\underset{\partial w_{i} P m}{f r}$ the results in Lemmas 3-6. It is easily seen that $\frac{\partial w_{i}^{S h *}}{\partial \varepsilon}>0, \frac{\partial w_{i}^{\prime l *}}{\partial \varepsilon}<0, \frac{\partial w_{i}^{P h *}}{\partial \varepsilon}>0$, and $\frac{\partial w_{i}^{P f}}{\partial \varepsilon}<0$ (see Appendices A-2 to A-5). Recall, from Sect. 5.1 that the manufacturers set higher wholesale prices when the distribution of the value-added services' cost is more dispersed. From the above inequalities, we notice that the manufacturers should set higher prices under more dispersed distributions of the value-added services only if the retailer's cost efficiency is high, i.e., $\mu \in U[\bar{\mu}-\varepsilon, \bar{\mu}]$. Moreover, if the retailer is relatively inefficient, i.e., $\mu \in U[\bar{\mu}, \bar{\mu}+\varepsilon]$, then the manufacturers should lower their wholesale prices when they are faced with more dispersed distributions of the value-added service cost. Surprisingly, this result is true under complete as well as partial information scenarios.

Next, we compare the profits of the retailer and manufacturers in various settings.

\section{Profit comparison}

In this section, we analyze each participant's profit under different scenarios of the retailer's information revelation mechanism. Moreover, we investigate the existence of an equilibrium in the first stage.

\subsection{Manufacturers' profits}

We compare the manufacturers' profits under different vertical information sharing decisions first. Recall that Manufacturer 1 is always the informed manufacturer under partial information sharing scenarios.

Proposition 1 For manufacturer 1, we have $\pi_{M 1}^{N} \geq \pi_{M 1}^{S}$ and $\pi_{M 1}^{N} \geq \pi_{M 1}^{P}$.

This proposition indicates that manufacturer 1 is always better off with no cost information sharing than with full information sharing or with partial information sharing. Thus, manufacturer 1 should not have any incentive to acquire or demand information as it may be detrimental to be informed in her case. This result is not consistent with previous research (Li, 2002; Yao et al., 2008).

Moreover, a comparison between the full and partial information sharing scenarios yields the following result.

Proposition 2 If $\mu \in U[\bar{\mu}-\varepsilon, \bar{\mu}]$, then $\pi_{M 1}^{S h} \geq \pi_{M 1}^{P h}$; if $\mu \in U[\bar{\mu}, \bar{\mu}+\varepsilon]$, then $\pi_{M 1}^{P l} \geq \pi_{M 1}^{S l}$. 
According to Proposition 2, when the cost efficiency is high, manufacturer 1 will be better off under the full information sharing scenario. However, when the cost efficiency is low, manufacturer 1 would prefer to be the only manufacturer in the market who has access to the private cost information of the retailer. Thus, if a manufacturer knows that the efficiency of the retailer regarding the cost associated with the valueadded services is low, then he should have an incentive to suppress this cost information from the other competing manufacturers in the market.

We now turn attention to manufacturer 2 .

Proposition 3 For manufacturer 2, we have $\pi_{M 2}^{N} \geq \pi_{M 2}^{S}$ and $\pi_{M 2}^{P} \geq \pi_{M 2}^{S}$.

This Proposition is interesting because manufacturer 2 is always worse off with complete cost information sharing; thus, manufacturer 2 prefers no vertical information sharing or partial information sharing.

Next, we compare manufacturer 2's profit under no vertical information sharing with that of partial information sharing.

Proposition 4 If $\mu \in U[\bar{\mu}-\varepsilon, \bar{\mu}]$, then $\pi_{M 2}^{P h} \geq \pi_{M 2}^{N}$; if $\mu \in U[\bar{\mu}, \bar{\mu}+\varepsilon]$, then $\pi_{M 2}^{N} \geq \pi_{M 2}^{P l}$.

According to Proposition 4, if the cost efficiency is high, then manufacturer 2 will be better off with partial information sharing. However, when the cost efficiency is low, manufacturer 2 will be better off with no vertical information sharing. Since cost-sharing provides additional information to manufacturer 1, it is quite natural for manufacturer 2 to be in a disadvantageous position under vertical cost-sharing. However, we find it interesting that manufacturer 2 will be in a favorable position under vertical cost-sharing when the cost efficiency is high. Note that, under cost-sharing, manufacturer 1 increases the wholesale price of product $\mathrm{A}$, thereby increasing the demand for product $\mathrm{B}$; this could be the indirect driver of our result under the high cost-efficiency.

\subsection{Retailer's profits}

We classify conditions under which the retailer has an incentive to reveal his private cost information to both manufacturers or partially to manufacturer 1 in this subsection. For convenience, we assume that $\alpha_{1}=\alpha_{2}=\alpha$ and $\beta_{1}=\beta_{2}=\beta$. When the retailer's cost efficiency is low, we can derive the following order relationship on profits.

Proposition 5 If $\mu \in U[\bar{\mu}, \bar{\mu}+\varepsilon]$, then $\pi_{R}^{S l} \geq \pi_{R}^{N} \geq \pi_{R}^{P l}$.

Thus, the best response of the retailer in the first stage is to share full information when the cost efficiency is low. Note that, when the cost efficiency is low, the manufacturers would announce lower wholesale prices under full information sharing scenario than those under no information sharing scenario, thereby increase the profit of the retailer. On the other hand, the retailer benefits more with no information sharing scenario over the partial information sharing scenario. This observation is interesting as intuitively we would expect the opposite. However, if the profit generated by product ' $A$ ' by reducing the retail price and increasing the channel demand is less than the extent of the losses caused by the decrease in the channel demand of product ' $\mathrm{B}$ ', then the retailer may be worse off by 
sharing information with only one manufacturer (thereby providing her unhealthy leverage in the market).

Proposition 6 If $\mu \in U[\bar{\mu}-\varepsilon, \bar{\mu}]$, then $\pi_{R}^{N} \geq \pi_{R}^{P h} \geq \pi_{R}^{S h}$.

In contrast, the retailer should prefer no information sharing scenario if the cost efficiency is high. Observe that, when the cost efficiency is high, the manufacturers should declare higher wholesale prices under full information sharing scenario than those under no information sharing scenario; this could have a detrimental effect on retailer's profit. Moreover, our results further suggest that if the retailer must reveal his private cost information as a result of a given market circumstance, even then he would prefer to share that information with only one manufacturer as opposed to sharing it with both the manufacturers.

To summarize, Propositions 5-6 highlight the role of the retailer's cost efficiency on his decisions with regard to sharing cost information with his suppliers. In particular, the retailer ought to take contrasting decisions under two cost efficiency settings-high and low.

Next, we examine further to see the best responses of the retailer, as describe in the two propositions above, indeed form unique equilibriums in the first stage. For this, we first consider the case where the retailer's cost efficiency is high, i.e., $\mu \in U[\bar{\mu}-\varepsilon, \bar{\mu}]$. From Proposition 6, we know that the retailer prefers to share no information in this case. Moreover, Proposition 1 indicates that manufacturer 1 also favors no information sharing in this case. Consequently, both the retailer and manufacturer 1 do not have any incentive to move away from the no information sharing scenario. However, according to Propositions 3-4, manufacturer 2 prefers partial information sharing when the retailer's cost efficiency is high. In order to check whether no information sharing scenario forms a unique equilibrium, in this case, we ask the following important question: Can manufacturer 2 motivate the retailer and manufacturer 1 (who are in the no information scenario) to move towards the partial information sharing $(P h)$ by introducing a side-payment? To answer this question, note that manufacturer 2 's gain by moving from scenario ' $N$ ' to ' $P h$ ' is $\left(\pi_{M 2}^{P h}-\pi_{M 2}^{N}\right)$, and the total loss incurred by the retailer and manufacturer 1 in the same course of action is $\left(\pi_{M 1}^{N}-\pi_{M 1}^{P h}\right)+\left(\pi_{R}^{N}-\pi_{R}^{P h}\right)$. It can be shown that $\left(\pi_{M 1}^{N}-\pi_{M 1}^{P h}\right)+\left(\pi_{R}^{N}-\pi_{R}^{P h}\right)>\left(\pi_{M 2}^{P h}-\pi_{M 2}^{N}\right)$ (see Appendix A-13). Consequently, the losses outweigh the gains in this case and manufacturer 2 will not be able to incentivize the other participants to move away from the no information sharing scenario. Thus, the unique equilibrium is indeed the no information sharing scenario in the first stage. We summarize our analysis above in the following proposition.

Proposition 7 If the retailer's cost efficiency is high, no information sharing is the unique equilibrium in the first stage of the game.

A parallel argument yields the following analogous result for the case with low-cost efficiency.

Proposition 8 If the retailer's cost efficiency is low, full information sharing is the unique equilibrium in the first stage of the game. 
Table 2 Manufacturers' profit under different service cost efficiency scenarios

\begin{tabular}{llllll}
\hline & $N$ & $S h$ & $S l$ & $P h$ & $P l$ \\
\hline$\pi_{M_{1}}$ & 1915.7 & 959.4884 & 956.1926 & 959.1830 & 956.4975 \\
$\pi_{M_{2}}$ & 1915.7 & 959.4884 & 956.1926 & 1916.3 & 1915.1 \\
\hline
\end{tabular}

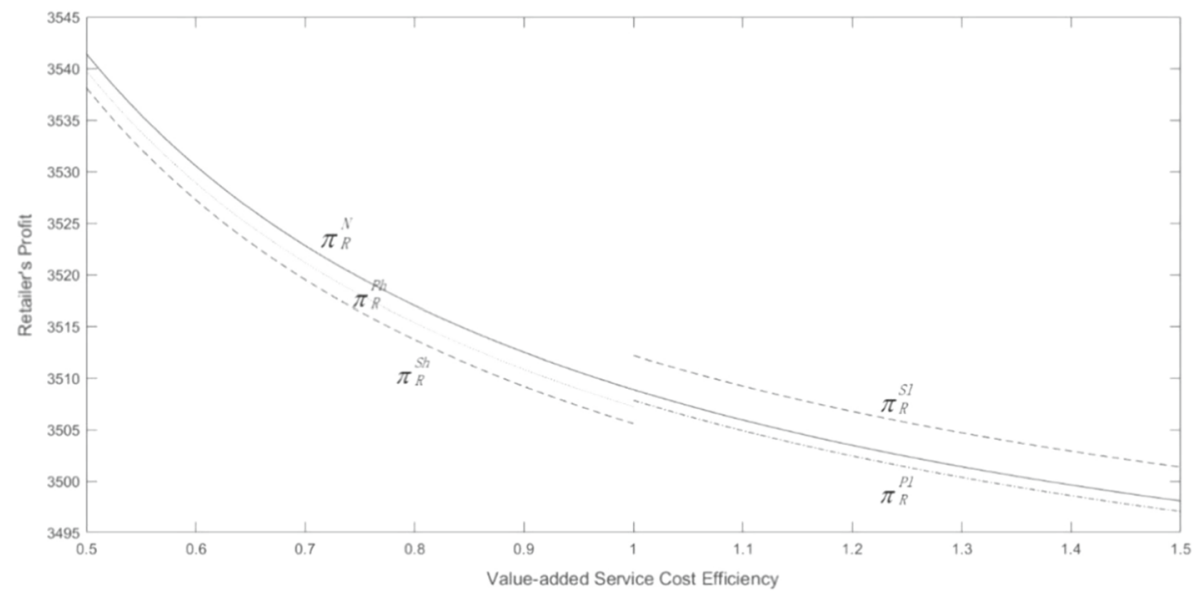

Fig. 3 The retailer's profit under different service cost efficiency scenarios

\subsection{Numerical results}

In this subsection, we present a numerical example to verify and enhance the understanding of the analytical results presented in the propositions in Sects. 6.1-6.2. The following parameter values are used in our example: $k=0.5, \bar{\mu}=1, \varepsilon=0.5, \alpha=\alpha_{1}=\alpha_{2}=100$ and $\beta=\beta_{1}=\beta_{2}=0.6$. Recall that $\mu \in[0.5,1]$ and $\mu \in[1,1.5]$ represent the high and low service cost efficiencies, respectively; moreover, we use $\mu_{1}=0.8$ and $\mu_{1}=1.2$ as representative values for each of these cases. The optimal profits of the manufacturers under different scenarios are presented in Table 2. Observe from Table 2 that two manufacturers obtain identical profits with full or no vertical information sharing irrespective of the cost efficiency of the retailer.

Observe further from Table 2 that $\left(\pi_{M 1}^{N}-\pi_{M 1}^{P h}\right)=956.2>0.6=\left(\pi_{M 2}^{P h}-\pi_{M 2}^{N}\right)$. Thus, the potential loss that will be incurred by manufacturer 1 by switching to partial $(P h)$ from no $(N)$ information sharing scenarios is much more than the profit gain of manufacturer 2 during the same course of action. Therefore, as we discussed in our analysis of the results in Proposition 7, manufacturer 2 would not be able to incentivize manufacturer 1 and/or retailer to deviate away from the 'no' information sharing scenario using a side payment.

Now, we turn attention to the retailer's profit. Figure 3 illustrates that the retailer's profit decreases as the cost efficiency coefficient $(\mu)$ increases. Thus, the retailer should do his best to improve the service cost efficiency in order to decrease his service cost as well as to stimulate channel demand and obtain more profit. From Fig. 3, we can see that if the service cost is efficient, i.e., $\mu \in[0.5,1]$, then the retailer's highest profit comes under the no vertical information sharing scenario; this observation is consistent with Proposition 6. 
Moreover, when the service cost efficiency is low, i.e., $\mu \in[1,1.5]$, the retailer performs well under the complete information sharing scenario, thereby confirming our results in Proposition 5.

Our numerical study also explains how the retailer's profit changes as the competition between products $\mathrm{A}$ and $\mathrm{B}$ characterized by $k$ increases. Here, we assume $\alpha=100, \beta=0.6$, $\bar{\mu}=1, \varepsilon=0.5$, and $\mu_{1}=1.2$. Figure 4 reveals that the retailer's profit increases as the competition between products $\mathrm{A}$ and $\mathrm{B}$ increases. It should be noted that while the profit increases under each scenario, they are not identical (see Fig. 7 in Appendix A-16). Consequently, according to our observation here, the retailer earns higher profits under more intense competition between the products.

In a similar manner, we can also investigate how the manufacturers' profit changes as the competition between products $\mathrm{A}$ and $\mathrm{B}$ characterized by $k$ increases. For that, we assume $\alpha_{1}=\alpha_{2}=100, \beta_{1}=\beta_{2}=0.6, \bar{\mu}=1, \varepsilon=0.5$. As seen in Figs. 5 and 6 below, manufacturers' profits decrease as the intensity of the competition between products increases.

\subsection{Revenue sharing contract}

We briefly introduce revenue-sharing contracts in this section and show that revenuesharing contracts coordinate the supply chain studied in this paper.

A revenue-sharing contract in a supply chain refers to a coordination method in which a retailer delivers a certain percentage of sales revenue to a supplier to obtain a lower wholesale price and improve the performance of the supply chain. This contract first appeared in the audiovisual rental industry and was later extended to other industries. In many consumer goods industries, suppliers increasingly adopt attractive wholesale pricing schemes to influence retailer's order and supply chain profit (Giri \& Sarker, 2016). Liu et al. (2018) study fairness effects in the logistics service supply chain when there is one logistics service integrator (LSI) and multiple functional logistics service providers (FLSPs). In their setting, both parties are traded by revenue sharing contracts; the LSI decides the revenue sharing portion and each FLS's perception of fairness depends on the final revenue allocation. They find that, when the demand is updated, the fairness preference behavior of the

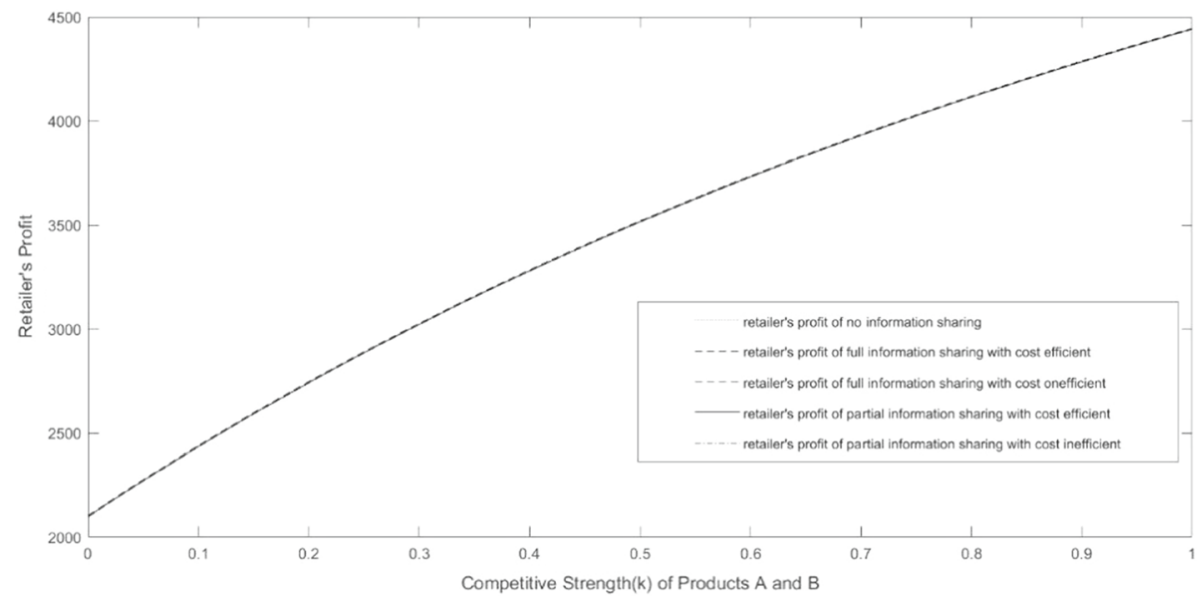

Fig. 4 The retailer's profit increases as the competition between products A and B characterized by $\mathrm{k}$ increases 


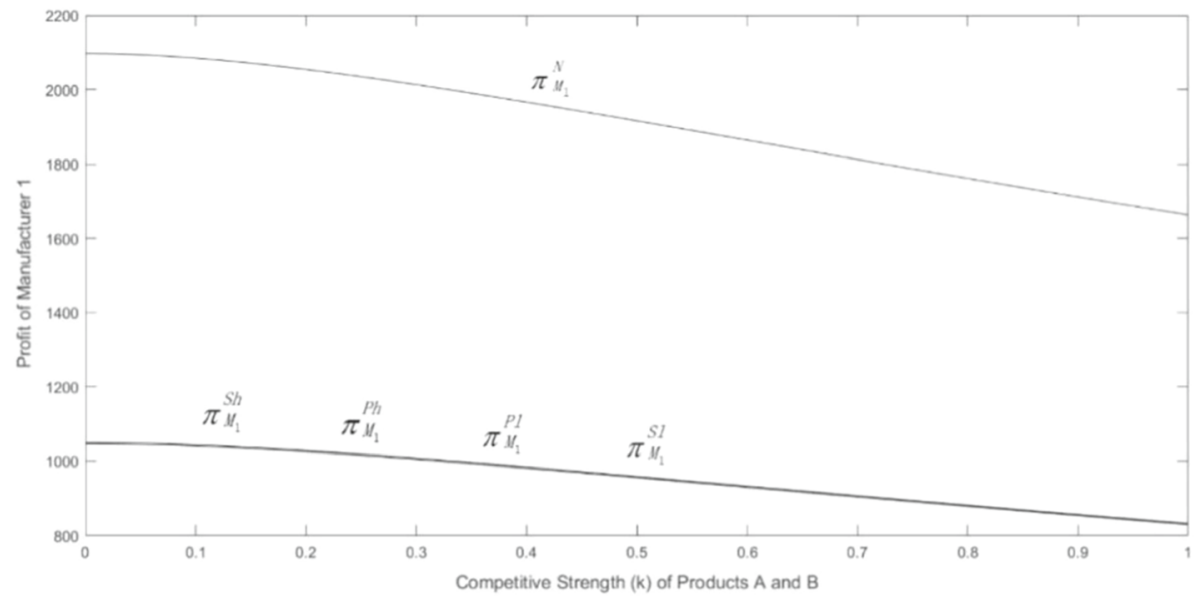

Fig. 5 Manufacturer 1's profit decreases as the competition between products A and B characterized by $\mathrm{k}$ increases

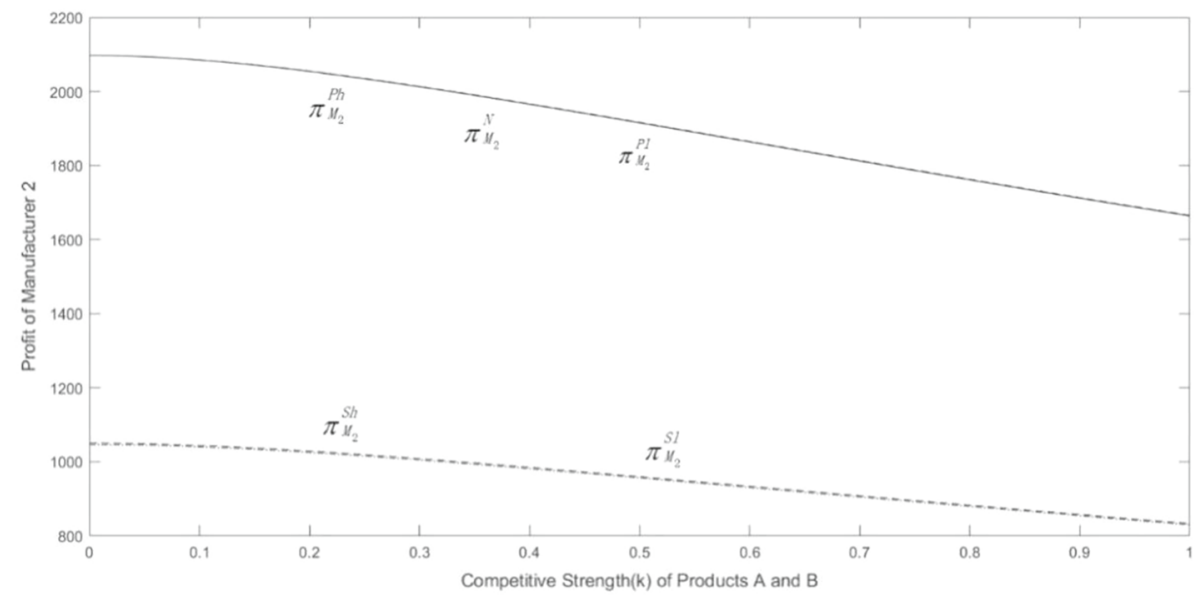

Fig. 6 Manufacturer 2's profit decreases as the competition between products A and B characterized by k increases

FLSPs affects their cooperation with the LSI, and that there exists an optimal updating time that maximizes supply chain performance. In another paper, Hu et al. (2017) consider a supplier that produces a product and sells it to market through a retailer by consignment contract. They focus on two popular consignment contracts-vendor managed consignment inventory contract and consignment contract with revenue sharing. Comparing analytical results between the two contracts, they find that the retailer and the whole supply chain benefit more from the consignment contract with revenue sharing. Motivated by these existing studies, we employ a classical revenue sharing contract (Shen et al., 2019) in our setting and identify the conditions under which the current supply chain is coordinated, 
i.e., $\pi_{S C}^{R S C} \geq \pi_{S C}^{N}$, where $\pi_{S C}^{R S C}$ denotes the total supply chain profit under the revenue sharing contract. These conditions are presented in the propositions below.

Proposition 9 Let $w_{1}^{N *}:=\frac{2 \beta_{1} \beta_{2}^{2} \ln \left(\frac{\bar{\mu}+\varepsilon}{\bar{\mu}-\varepsilon}\right)+3 \beta_{2}^{2} k \ln \left(\frac{\bar{\mu}+\varepsilon}{\bar{\mu}-\varepsilon}\right)+8 \alpha_{1} \beta_{1}^{2} \varepsilon+8 \alpha_{1} \beta_{1} k \varepsilon+4 \alpha_{2} \beta_{1} k \varepsilon}{4 \beta_{1} \varepsilon\left(4 \beta_{1}^{2}+8 \beta_{1} k+3 k^{2}\right)}, c:=\frac{3 \beta_{2}^{2}}{2 \beta_{1}^{2} \mu_{1}}-\frac{3 \beta_{1} \beta_{2}^{2}}{4\left(\beta_{1}+k\right)}$, and $g:=\frac{k}{\beta_{1}+k}$. Then, when the value-added service cost efficiency is high, $\frac{3 \beta_{2}^{2}\left(1-\beta_{1}^{4} \mu_{1}^{2}\right)}{2 \beta_{1}^{2} \mu_{1}} \leq w_{1} \leq w_{1}^{N *}$ and $c+g w_{1} \leq w_{2} \leq \frac{w_{1}-c}{g}$, we have that $\pi_{S C}^{R S C} \geq \pi_{S C}^{N}$.

Proposition 10 Let $w_{1}^{S l *}:=\frac{2 \beta_{1} \beta_{2}^{2} \ln \left(\frac{\bar{\mu}+\varepsilon}{\bar{\mu}}\right)+3 \beta_{2}^{2} k \ln \left(\frac{\bar{\mu}+\varepsilon}{\bar{\mu}}\right)+4 \alpha_{1} \beta_{1}^{2} \varepsilon+4 \alpha_{1} \beta_{1} k \varepsilon+2 \alpha_{2} \beta_{1} k \varepsilon}{2 \beta_{1} \varepsilon\left(4 \beta_{1}^{2}+8 \beta_{1} k+3 k^{2}\right)}$, $c:=\frac{3 \beta_{2}^{2}}{2 \beta_{1}^{2} \mu_{1}}-\frac{3 \beta_{1} \beta_{2}^{2}}{4\left(\beta_{1}+k\right)}$, and $g:=\frac{k}{\beta_{1}+k}$, Then, when the value-added service cost efficiency is low, $\frac{3 \beta_{2}^{2}\left(1-\beta_{1}^{4} \mu_{1}^{2}\right)}{2 \beta_{1}^{2} \mu_{1}} \leq w_{1} \leq w_{1}^{S l *}$ and $c+g w_{1} \leq w_{2} \leq \frac{w_{1}-c}{g}$, we have that $\pi_{S C}^{R S C} \geq \pi_{S C}^{N}$.

\section{Conclusions and further research}

In this paper, we have studied how the retailer's decisions on sharing cost information about the value-added services are made and how his decision impacts the decisions of the manufacturers in a supply chain with a common retailer and two manufacturers. We consider five scenarios based upon the retailer's cost efficiency-high $(h)$ or low $(l)$ and information sharing setup- $S, N$, and $P$. Using a three-stage game-theoretic model, we derive optimal pricing strategies for each player as well as the optimal level for valueadded services and information sharing strategies for the retailer in the first stage.

Our results suggest that the optimal level for value-added services is mainly determined by the retailer's service cost efficiency. Moreover, based on our findings, information sharing does not always create a win-win situation in a supply chain. Specifically, we find that if the value-added service cost efficiency is high, i.e., $\mu \in U[\bar{\mu}-\varepsilon, \bar{\mu}]$, then the retailer and manufacturer 1 are better off with no vertical information sharing while manufacturer 2 prefers partial information sharing; furthermore, manufacturer 2 is unable to motivate other participants to accept partial information sharing through a side payment. In contrast, when the service cost efficiency is low, i.e., $\mu \in U[\bar{\mu}, \bar{\mu}+\varepsilon]$, two manufacturers are better off with no vertical information sharing while the retailer prefers to share complete information with them. We also find that manufacturer 1 is always better off with no information sharing whereas manufacturer 2 is always worse off with complete cost information sharing. Finally, our numerical study reveals that the impact of competitive strengths on the profit of the two manufacturers and the retailer is completely different. In other words, the retailer's and manufacturers' profit respectively increases and decreases as the competition between products increases. In summary, this study complements the existing supply chain literature on the value-added service cost information sharing and our research findings should help supply chain firms to make better decisions.

Although our study provides useful insights for supply chain firms, it is possible to further enrich our contribution in a possible future study. For example, we assume that the cost efficiency is uniformly distributed. However, it would be a challenging exercise to see if our results hold under other popular probability distributions. Also, one could study the impact of horizontal information leakage between the two manufacturers in our setting. Finally, it would be interesting to see how our results going to change in a setting with 
multiple retailers where different retailers provide different levels of value-added services to products.

\section{Appendices}

\section{A-1. Proof of Lemma 1}

For the retailer, for $i=1,2$, we have

$$
\underset{p_{i}, v}{\operatorname{Max}} E\left[\pi_{R} \mid w_{i}\right]=\underset{p_{i}, v}{\operatorname{Max}} E\left[\left(p_{1}-w_{1}-c\right) d_{1}+\left(p_{2}-w_{2}-c\right) d_{2} \mid w_{i}\right]
$$

From the first-order condition (FOC), we have the optimal response function for the retailer:

$$
\frac{\partial E\left[\pi_{R}\right]}{\partial P_{1}}=0, \quad \frac{\partial E\left[\pi_{R}\right]}{\partial P_{2}}=0, \quad \frac{\partial E\left[\pi_{R}\right]}{\partial V}=0 .
$$

The Hessian matrix is:

$$
H=\left[\begin{array}{ll}
\frac{\partial_{\pi_{R}}^{2}}{\partial p_{1}^{2}} & \frac{\partial_{\pi_{R}}^{2}}{\partial p_{1} p_{2}} \\
\frac{\partial_{\pi_{R}}^{2}}{\partial p_{2} p_{1}} & \frac{\partial_{\pi_{R}}^{2}}{\partial p_{2}^{2}}
\end{array}\right]=\left[\begin{array}{ll}
-2 \beta_{1}-2 k & 2 k \\
2 k & -2 \beta_{1}-2 k
\end{array}\right],
$$

and $|H|=\left(-2 \beta_{1}-2 k\right)^{2}-(2 k)^{2}=4 \beta_{1}^{2}+8 \beta_{1} k>0$. Thus, it is easily seen that the Hessian matrix $\mathrm{H}$ is a diagonally dominant matrix, thereby guaranteeing the joint concavity of the profit function $\pi_{R}\left(p_{1}, p_{2}\right)$. Consequently, we have

$$
\begin{gathered}
\mathrm{p}_{1}^{*}=\frac{\beta_{1} \mu_{1} v^{2}+2 \beta_{2} v+2 \alpha_{1}+2 \beta_{1} w_{1}+4 k p_{2}+2 k w_{1}-2 k w_{2}}{4\left(\beta_{1}+k\right)}, \\
\mathrm{p}_{2}^{*}=\frac{\beta_{1} \mu_{1} v^{2}+2 \beta_{2} v+2 \alpha_{2}+2 \beta_{1} w_{2}+4 k p_{1}-2 k w_{1}+2 k w_{2}}{4\left(\beta_{1}+k\right)}, \\
v^{*}=\frac{\beta_{2}}{\beta_{1} \mu_{1}} .
\end{gathered}
$$

Equation (17) is the best response function of the retailer for product A, which is affected by the retail price of product B. Then, combining (17)-(19), we can obtain that

$$
\mathrm{p}_{1}^{*}=\frac{3 \beta_{1} \beta_{2}^{2}+6 \beta_{2}^{2} k+2 \beta_{1}^{3} \mu_{1} w_{1}+2 \alpha_{1} \beta_{1}^{2} \mu_{1}+2 \alpha_{1} \beta_{1} k \mu_{1}+2 \alpha_{2} \beta_{1} k \mu_{1}+4 \beta_{1}^{2} k \mu_{1} w_{1}}{4 \beta_{1}^{2} \mu_{1}\left(\beta_{1}+2 k\right)} .
$$

Similarly, combining (18)—(20), we get

$$
\mathrm{p}_{2}^{*}=\frac{3 \beta_{1} \beta_{2}^{2}+6 \beta_{2}^{2} k+2 \beta_{1}^{3} \mu_{1} w_{2}+2 \alpha_{2} \beta_{1}^{2} \mu_{1}+2 \alpha_{1} \beta_{1} k \mu_{1}+2 \alpha_{2} \beta_{1} k \mu_{1}+4 \beta_{1}^{2} k \mu_{1} w_{2}}{4 \beta_{1}^{2} \mu_{1}\left(\beta_{1}+2 k\right)} .
$$




\section{A-2. Proof of Lemma 2}

For manufacturer 1, Eq. (9) can be simplified as

$$
\underset{w}{\operatorname{Max}} E\left[\pi_{M_{1}}\right]=\alpha_{1} \varepsilon w_{1}-\varepsilon k w_{1}^{2}-\beta_{1} \varepsilon w_{1}^{2}+\varepsilon k w_{1} w_{2}-\frac{\beta_{2}^{2} w_{1}(\ln (\bar{\mu}-\varepsilon)-\ln (\bar{\mu}+\varepsilon))}{4 \beta_{1}} .
$$

For manufacturer 2, we have

$$
\underset{w}{\operatorname{Max}} E\left[\pi_{M_{2}}\right]=\alpha_{2} \varepsilon w_{2}-\varepsilon k w_{2}^{2}-\beta_{1} \varepsilon w_{2}^{2}+\varepsilon k w_{1} w_{2}-\frac{\beta_{2}^{2} w_{2}(\ln (\bar{\mu}+\varepsilon)-\ln (\bar{\mu}-\varepsilon))}{4 \beta_{1}} .
$$

Since $\frac{\partial_{\pi_{M i}}^{2}}{\partial w_{i}^{2}}=-2 \beta_{1} \varepsilon-2 \varepsilon k<0, \pi_{M_{i}}$ is concave in $w_{i}$. Thus, setting $\frac{\partial E\left[\pi_{M i}\right]}{\partial W_{i}}=0$, we obtain the following optimal wholesale prices under no information sharing scenario:

$$
\begin{aligned}
& w_{1}^{N *}=\frac{2 \beta_{1} \beta_{2}^{2} \ln \left(\frac{\bar{\mu}+\varepsilon}{\bar{\mu}-\varepsilon}\right)+3 \beta_{2}^{2} k \ln \left(\frac{\bar{\mu}+\varepsilon}{\bar{\mu}-\varepsilon}\right)+8 \alpha_{1} \beta_{1}^{2} \varepsilon+8 \alpha_{1} \beta_{1} k \varepsilon+4 \alpha_{2} \beta_{1} k \varepsilon}{4 \beta_{1} \varepsilon\left(4 \beta_{1}^{2}+8 \beta_{1} k+3 k^{2}\right)}, \\
& w_{2}^{N *}=\frac{2 \beta_{1} \beta_{2}^{2} \ln \left(\frac{\bar{\mu}+\varepsilon}{\bar{\mu}-\varepsilon}\right)+3 \beta_{2}^{2} k \ln \left(\frac{\bar{\mu}+\varepsilon}{\bar{\mu}-\varepsilon}\right)+8 \alpha_{2} \beta_{1}^{2} \varepsilon+8 \alpha_{2} \beta_{1} k \varepsilon+4 \alpha_{1} \beta_{1} k \varepsilon}{4 \beta_{1} \varepsilon\left(4 \beta_{1}^{2}+8 \beta_{1} k+3 k^{2}\right)} .
\end{aligned}
$$

It is easily seen that $\frac{\partial w_{i}^{N *}}{\partial \varepsilon}=\frac{\beta_{2}^{2}\left(2 \beta_{1}+3 k\right)\left[\varepsilon^{2} \ln \left(\frac{\bar{\mu}+\varepsilon}{\bar{\mu}-\varepsilon}\right)-\bar{\mu}^{2} \ln \left(\frac{\bar{\mu}+\varepsilon}{\bar{\mu}-\varepsilon}\right)+2 \varepsilon \bar{\mu}\right]}{4 \beta_{1} \varepsilon^{2}\left(\bar{\mu}^{2}-\varepsilon^{2}\right)\left(4 \beta_{1}^{2}+8 \beta_{1} k+3 k^{2}\right)}$. Since $(\bar{\mu}-\varepsilon)>0$, we have that $\left(\bar{\mu}^{2}-\varepsilon^{2}\right)>0$. Next, let

$$
\Upsilon(\varepsilon)=\varepsilon^{2} \ln \left(\frac{\bar{\mu}+\varepsilon}{\bar{\mu}-\varepsilon}\right)-\bar{\mu}^{2} \ln \left(\frac{\bar{\mu}+\varepsilon}{\bar{\mu}-\varepsilon}\right)+2 \varepsilon \bar{\mu} .
$$

Then, $\frac{\partial \Upsilon(\varepsilon)}{\partial \varepsilon}=2 \varepsilon \ln \frac{\bar{\mu}+\varepsilon}{\bar{\mu}-\varepsilon}>0$, and $\Upsilon(0)=0$. Hence $\Upsilon(\varepsilon)>0$ for all $\varepsilon>0$. Thus, $\frac{\partial w_{i}^{N *}}{\partial \varepsilon}>0$.

\section{A-3. Proof of Lemma 3}

For manufacturer 1, Eq. (10) can be simplified as

$$
\underset{w}{\operatorname{Max}} E\left[\pi_{M_{1}}\right]=\frac{\alpha_{2} \varepsilon w_{1}}{2}+\frac{\varepsilon k w_{1} w_{2}}{2}-\frac{\beta_{1} \varepsilon w_{1}^{2}}{2}-\frac{\varepsilon k w_{1}^{2}}{2}+\frac{\beta_{2}^{2} w_{1}(\ln (\bar{\mu})-\ln (\bar{\mu}-\varepsilon))}{4 \beta_{1}}
$$

Moreover, for manufacturer 2, we have

$$
\underset{w}{\operatorname{Max}} E\left[\pi_{M_{2}}\right]=\frac{\alpha_{2} \varepsilon w_{2}}{2}+\frac{\varepsilon k w_{1} w_{2}}{2}-\frac{\beta_{1} \varepsilon w_{2}^{2}}{2}-\frac{\varepsilon k w_{2}^{2}}{2}+\frac{\beta_{2}^{2} w_{2}(\ln (\bar{\mu})-\ln (\bar{\mu}-\varepsilon))}{4 \beta_{1}}
$$

Since $\frac{\partial_{\pi_{M i}}^{2}}{\partial w_{i}^{2}}=-2 \beta_{1} \varepsilon-2 \varepsilon k<0, \pi_{M_{i}}$ is concave in $w_{i}$, using $\frac{\partial E\left[\pi_{M i}\right]}{\partial W_{i}}=0$, we obtain the optimal wholesale prices under full information sharing scenario as: 


$$
\begin{aligned}
& w_{1}^{S h *}=\frac{2 \beta_{1} \beta_{2}^{2} \ln \left(\frac{\bar{\mu}}{\bar{\mu}-\varepsilon}\right)+3 \beta_{2}^{2} k \ln \left(\frac{\bar{\mu}}{\bar{\mu}-\varepsilon}\right)+4 \alpha_{1} \beta_{1}^{2} \varepsilon+4 \alpha_{1} \beta_{1} k \varepsilon+2 \alpha_{2} \beta_{1} k \varepsilon}{2 \beta_{1} \varepsilon\left(4 \beta_{1}^{2}+8 \beta_{1} k+3 k^{2}\right)}, \\
& w_{2}^{S h *}=\frac{2 \beta_{1} \beta_{2}^{2} \ln \left(\frac{\bar{\mu}}{\bar{\mu}-\varepsilon}\right)+3 \beta_{2}^{2} k \ln \left(\frac{\bar{\mu}}{\bar{\mu}-\varepsilon}\right)+4 \alpha_{2} \beta_{1}^{2} \varepsilon+2 \alpha_{1} \beta_{1} k \varepsilon+4 \alpha_{2} \beta_{1} k \varepsilon}{2 \beta_{1} \varepsilon\left(4 \beta_{1}^{2}+8 \beta_{1} k+3 k^{2}\right)} .
\end{aligned}
$$

Moreover, $\frac{\partial W_{i}^{N *}}{\partial \varepsilon}=\frac{-\beta_{2}^{2}\left[\varepsilon-\bar{\mu} \ln \left(\frac{\bar{\mu}}{\bar{\mu}-\varepsilon}\right)+\varepsilon \ln \left(\frac{\bar{\mu}}{\bar{\mu}-\varepsilon}\right)\right]}{2 \beta_{1} \varepsilon^{2}(\bar{\mu}-\varepsilon)\left(2 \beta_{1}+k\right)}$. Since $(\bar{\mu}-\varepsilon)>0$, in order to see $\frac{\partial w_{i}^{N *}}{\partial \varepsilon}<0$, we only need to show that

$$
\Theta(\varepsilon):=\varepsilon-\bar{\mu} \ln \left(\frac{\bar{\mu}}{\bar{\mu}-\varepsilon}\right)+\varepsilon \ln \left(\frac{\bar{\mu}}{\bar{\mu}-\varepsilon}\right)>0 .
$$

For this, note that $\frac{\partial \Theta(\varepsilon)}{\partial \varepsilon}=\ln \frac{\bar{\mu}}{\bar{\mu}-\varepsilon}>0$. Then, since $\Theta(0)=0$, we have that $\Theta(\varepsilon)>0$.

\section{A-4. Proof of Lemma 4}

This proof is similar to that of Lemma 3.

\section{A-5. Proof of Lemma 5}

For manufacturer 1, Eq. (12) can be simplified as

$$
\underset{w}{\operatorname{Max}} E\left[\pi_{M_{1}}\right]=\frac{\alpha_{1} \varepsilon w_{1}}{2}+\frac{\varepsilon k w_{1} w_{2}}{2}-\frac{\beta_{1} \varepsilon w_{1}^{2}}{2}-\frac{\varepsilon k w_{1}^{2}}{2}+\frac{\beta_{2}^{2} w_{1}(\ln (\bar{\mu})-\ln (\bar{\mu}-\varepsilon))}{4 \beta_{1}} .
$$

Also, for manufacturer 2, Eq. (13) can be simplified as

$$
\underset{w}{\operatorname{Max}} E\left[\pi_{M_{2}}\right]=\alpha_{2} \varepsilon w_{2}-\varepsilon k w_{2}^{2}-\beta_{1} \varepsilon w_{2}^{2}+\varepsilon k w_{1} w_{2}+\frac{\beta_{2}^{2} w_{2}(\ln (\bar{\mu})-\ln (\bar{\mu}-\varepsilon))}{4 \beta_{1}} .
$$

Note that $\frac{\partial_{\pi_{M_{i}}}^{2}}{\partial w_{i}^{2}}=-2 \beta_{1} \varepsilon-2 \varepsilon k<0$; thus, $\pi_{M_{i}}$ is concave in $w_{i}$. Therefore, $\frac{\partial E\left[\pi_{M i}\right]}{\partial w_{i}}=0$ gives the following optimal wholesale prices under the full information sharing scenario.

$$
\begin{aligned}
& w_{1}^{P h *}=\frac{4 \beta_{1} \beta_{2}^{2} \ln \left(\frac{\bar{\mu}}{\bar{\mu}-\varepsilon}\right)+4 \beta_{2}^{2} k \ln \left(\frac{\bar{\mu}}{\bar{\mu}-\varepsilon}\right)+\beta_{2}^{2} k \ln \left(\frac{\bar{\mu}+\varepsilon}{\bar{\mu}-\varepsilon}\right)+8 \alpha_{1} \beta_{1}^{2} \varepsilon+8 \alpha_{1} \beta_{1} k \varepsilon+2 \alpha_{2} \beta_{1} k \varepsilon}{4 \beta_{1} \varepsilon\left(4 \beta_{1}^{2}+8 \beta_{1} k+3 k^{2}\right)}, \\
& w_{2}^{P h *}=\frac{\beta_{1} \beta_{2}^{2} \ln \left(\frac{\bar{\mu}+\varepsilon}{\bar{\mu}-\varepsilon}\right)+\beta_{2}^{2} k \ln \left(\frac{\bar{\mu}}{\bar{\mu}-\varepsilon}\right)+\beta_{2}^{2} k \ln \left(\frac{\bar{\mu}+\varepsilon}{\bar{\mu}-\varepsilon}\right)+4 \alpha_{2} \beta_{1}^{2} \varepsilon+2 \alpha_{1} \beta_{1} k \varepsilon+4 \alpha_{2} \beta_{1} k \varepsilon}{2 \beta_{1} \varepsilon\left(4 \beta_{1}^{2}+8 \beta_{1} k+3 k^{2}\right)} .
\end{aligned}
$$

Moreover, we can get that 


$$
\begin{aligned}
& \frac{\partial W_{i}^{P h *}}{\partial \varepsilon}=\frac{\beta_{2}^{2}\left[\varepsilon^{2} k+\beta_{1} \varepsilon^{2} \ln \left(\frac{\bar{\mu}+\varepsilon}{\bar{\mu}-\varepsilon}\right)+\varepsilon^{2} k \ln \left(\frac{\bar{\mu}+\varepsilon}{\bar{\mu}-\varepsilon}\right)-\beta_{1} \bar{\mu}^{2} \ln \left(\frac{\bar{\mu}+\varepsilon}{\bar{\mu}-\varepsilon}\right)+2 \beta_{1} \varepsilon \bar{\mu}-k \bar{\mu}^{2} \ln \left(\frac{\bar{\mu}+\varepsilon}{\bar{\mu}-\varepsilon}\right)+3 k \varepsilon \bar{\mu}+\varepsilon^{2} k \ln \left(\frac{\bar{\mu}}{\bar{\mu}-\varepsilon}\right)-k \bar{\mu}^{2} \ln \left(\frac{\bar{\mu}+\varepsilon}{\bar{\mu}-\varepsilon}\right)\right]}{2 \beta_{1} \varepsilon^{2}\left(\bar{\mu}^{2}-\varepsilon^{2}\right)\left(4 \beta_{2}^{2}+3 k^{2}+8 \beta_{1} k\right)} \\
& \text { Let } \quad \Psi(\varepsilon):=\varepsilon^{2} k+\beta_{1} \varepsilon^{2} \ln \left(\frac{\bar{\mu}+\varepsilon}{\bar{\mu}-\varepsilon}\right)+\varepsilon^{2} k \ln \left(\frac{\bar{\mu}+\varepsilon}{\bar{\mu}-\varepsilon}\right)-\beta_{1} \bar{\mu}^{2} \ln \left(\frac{\bar{\mu}+\varepsilon}{\bar{\mu}-\varepsilon}\right)+2 \beta_{1} \varepsilon \bar{\mu}-k \bar{\mu}^{2} \ln \\
& \left(\frac{\bar{\mu}+\varepsilon}{\bar{\mu}-\varepsilon}\right)+3 k \varepsilon \bar{\mu}+\varepsilon^{2} k \ln \left(\frac{\bar{\mu}}{\bar{\mu}-\varepsilon}\right)-k \bar{\mu}^{2} \ln \left(\frac{\bar{\mu}+\varepsilon}{\bar{\mu}-\varepsilon}\right), \quad \text { Then, we have } \\
& \frac{\partial \Psi(\varepsilon)}{\partial \varepsilon}=\varepsilon\left(k+2 k \ln \frac{\bar{\mu}}{\bar{\mu}-\varepsilon}+2 \beta_{1} \ln \frac{\bar{\mu}+\varepsilon}{\bar{\mu}-\varepsilon}+2 k \ln \frac{\bar{\mu}+\varepsilon}{\bar{\mu}-\varepsilon}\right)>0 \text {. Since } \Psi(0)=0 \text {, we can deduce that } \\
& \Psi(\varepsilon)>0 \text { for all } \varepsilon>0 \text {. Finally, since }\left(\bar{\mu}^{2}-\varepsilon^{2}\right)>0 \text {, we obtain that } \frac{\partial W_{i}^{P h *}}{\partial \varepsilon}>0 \text {. }
\end{aligned}
$$

\section{A-6. Proof of Lemma 6}

This proof is similar to that of Lemma 5 .

\section{A-7. Proof of Proposition 1}

Substituting the optimal prices in profit expressions, we can derive that

$$
\begin{aligned}
& \pi_{M 1}^{N}=\frac{\left(\beta_{1}+k\right)\left[2 \beta_{1} \beta_{2}^{2} \ln \left(\frac{\bar{\mu}+\varepsilon}{\bar{\mu}-\varepsilon}\right)+3 \beta_{2}^{2} k \ln \left(\frac{\bar{\mu}+\varepsilon}{\bar{\mu}-\varepsilon}\right)+8 \alpha_{1} \beta_{1}^{2} \varepsilon+8 \alpha_{1} \beta_{1} \varepsilon k+4 \alpha_{2} \beta_{1} \varepsilon k\right]^{2}}{16 \beta_{1}^{2} \varepsilon\left(4 \beta_{1}^{2}+8 \beta_{1} k+3 k^{2}\right)^{2}}, \\
& \pi_{M 1}^{S h}=\frac{\left(\beta_{1}+k\right)\left[2 \beta_{1} \beta_{2}^{2} \ln \left(\frac{\bar{\mu}}{\bar{\mu}-\varepsilon}\right)+3 \beta_{2}^{2} k \ln \left(\frac{\bar{\mu}}{\bar{\mu}-\varepsilon}\right)+4 \alpha_{1} \beta_{1}^{2} \varepsilon+4 \alpha_{1} \beta_{1} \varepsilon k+2 \alpha_{2} \beta_{1} \varepsilon k\right]^{2}}{8 \beta_{1}^{2} \varepsilon\left(4 \beta_{1}^{2}+8 \beta_{1} k+3 k^{2}\right)^{2}}, \\
& \pi_{M 1}^{S l}=\frac{\left(\beta_{1}+k\right)\left[2 \beta_{1} \beta_{2}^{2} \ln \left(\frac{\bar{\mu}+\varepsilon}{\bar{\mu}}\right)+3 \beta_{2}^{2} k \ln \left(\frac{\bar{\mu}+\varepsilon}{\bar{\mu}}\right)+4 \alpha_{1} \beta_{1}^{2} \varepsilon+4 \alpha_{1} \beta_{1} \varepsilon k+2 \alpha_{2} \beta_{1} \varepsilon k\right]_{1}^{2} \varepsilon\left(4 \beta_{1}^{2}+8 \beta_{1} k+3 k^{2}\right)^{2}}{32 \beta_{1}^{2} \varepsilon\left(4 \beta_{1}^{2}+8 \beta_{1} k+3 k^{2}\right)^{2}}, \\
& \pi_{M 1}^{P h}=\frac{\left.\left(\beta_{1}+k\right)\left[4 \beta_{1} \beta_{2}^{2} \ln \left(\frac{\bar{\mu}}{\bar{\mu}-\varepsilon}\right)+4 \beta_{2}^{2} k \ln \left(\frac{\bar{\mu}}{\bar{\mu}-\varepsilon}\right)+\beta_{2}^{2} k \ln \left(\frac{\bar{\mu}+\varepsilon}{\bar{\mu}-\varepsilon}\right)+8 \alpha_{1} \beta_{1}^{2} \varepsilon+8 \alpha_{1} \beta_{1} \varepsilon k+4 \alpha_{2} \beta_{1} \varepsilon k\right]_{1}^{2} k+3 k^{2}\right)^{2}}{\left.36 \alpha_{1} \beta_{1}^{2} \varepsilon+8 \alpha_{1} \beta_{1} \varepsilon k+4 \alpha_{2} \beta_{1} \varepsilon k\right]^{2}} . \\
& \pi_{M 1}^{P l}=\frac{\left(\beta_{1}+k\right)\left[4 \beta_{1} \beta_{2}^{2} \ln \left(\frac{\bar{\mu}+\varepsilon}{\bar{\mu}}\right)+4 \beta_{2}^{2} k \ln \left(\frac{\bar{\mu}+\varepsilon}{\bar{\mu}}\right)+\beta_{2}^{2} k \ln \left(\frac{\bar{\mu}+\varepsilon}{\bar{\mu}-\varepsilon}\right)+\right.}{37)}
\end{aligned}
$$

First, some basic algebraic comparison of the profit expressions yields $\pi_{M 1}^{S h} \geq \pi_{M 1}^{S l}$.

Now let $\pi_{M 1}^{N}:=\frac{\left(\beta_{1}+k\right) A_{1}^{2}}{16 \beta_{1}^{2} \varepsilon\left(4 \beta_{1}^{2}+8 \beta_{1} k+3 k^{2}\right)^{2}}$ and $\pi_{M 1}^{S h}:=\frac{\left(\beta_{1}+k\right) B_{1}^{2}}{16 \beta_{1}^{2} \varepsilon\left(4 \beta_{1}^{2}+8 \beta_{1} k+3 k^{2}\right)^{2}}$, 
where

$$
\begin{aligned}
& A_{1}=2 \beta_{1} \beta_{2}^{2} \ln \left(\frac{\bar{\mu}}{\bar{\mu}-\varepsilon}\right)+3 \beta_{2}^{2} k \ln \left(\frac{\bar{\mu}}{\bar{\mu}-\varepsilon}\right)+8 \alpha_{1} \beta_{1}^{2} \varepsilon+8 \alpha_{1} \beta_{1} \varepsilon k+4 \alpha_{2} \beta_{1} \varepsilon k, \quad \text { and } \\
& B_{1}=\sqrt{2}\left[2 \beta_{1} \beta_{2}^{2} \ln \left(\frac{\bar{\mu}}{\bar{\mu}-\varepsilon}\right)+3 \beta_{2}^{2} k \ln \left(\frac{\bar{\mu}}{\bar{\mu}-\varepsilon}\right)+4 \alpha_{1} \beta_{1}^{2} \varepsilon+4 \alpha_{1} \beta_{1}^{2} \varepsilon k+2 \alpha_{2} \beta_{1} \varepsilon k\right] .
\end{aligned}
$$

It is easy to see that for sufficiently large $\alpha_{i}(i=1,2), A_{1} \geq B_{1}$. Thus, $\pi_{M 1}^{N} \geq \pi_{M 1}^{S h}$. So, we obtain the desired result, i.e., $\pi_{M 1}^{N} \geq \pi_{M 1}^{S}$.

Again, for the second part, it is an easy exercise to see that $\pi_{M 1}^{P h} \geq \pi_{M 1}^{P l}$.

Let $\pi_{M_{1}}^{N}:=\frac{\left(\beta_{1}+k\right) C_{1}^{2}}{32 \beta_{1}^{2} \varepsilon\left(4 \beta_{1}^{2}+8 \beta_{1} k+3 k^{2}\right)^{2}}$ and $\pi_{M_{1}}^{P h}:=\frac{\left(\beta_{1}+k\right) D_{1}^{2}}{32 \beta_{1}^{2} \varepsilon\left(4 \beta_{1}^{2}+8 \beta_{1} k+3 k^{2}\right)^{2}}$,

where

$$
\begin{aligned}
& C_{1}=\sqrt{2}\left[2 \beta_{1} \beta_{2}^{2} \ln \left(\frac{\bar{\mu}+\varepsilon}{\bar{\mu}-\varepsilon}\right)+3 \beta_{2}^{2} k \ln \left(\frac{\bar{\mu}+\varepsilon}{\bar{\mu}-\varepsilon}\right)+8 \alpha_{1} \beta_{1}^{2} \varepsilon+8 \alpha_{1} \beta_{1} \varepsilon k+4 \alpha_{2} \beta_{1} \varepsilon k\right], \quad \text { and } \\
& D_{1}=4 \beta_{1} \beta_{2}^{2} \ln \left(\frac{\bar{\mu}}{\bar{\mu}-\varepsilon}\right)+4 \beta_{2}^{2} k \ln \left(\frac{\bar{\mu}}{\bar{\mu}-\varepsilon}\right)+\beta_{2}^{2} k \ln \left(\frac{\bar{\mu}+\varepsilon}{\bar{\mu}-\varepsilon}\right)+8 \alpha_{1} \beta_{1}^{2} \varepsilon+8 \alpha_{1} \beta_{1} \varepsilon k+4 \alpha_{2} \beta_{1} \varepsilon k .
\end{aligned}
$$

Assuming that $\alpha_{i}$ is large enough, it is easy to conclude that $C_{1}>D_{1}$, implying that $\pi_{M 1}^{N} \geq \pi_{M 1}^{P h}$. Thus, we have that $\pi_{M 1}^{N} \geq \pi_{M 1}^{P}$.

\section{A-8. Proof of Proposition 2}

For the first part, let $\pi_{M 1}^{S h}:=\frac{\left(\beta_{1}+k\right) A_{2}^{2}}{32 \beta_{1}^{2} \varepsilon\left(4 \beta_{1}^{2}+8 \beta_{1} k+3 k^{2}\right)^{2}}$ and $\pi_{M 1}^{P h}:=\frac{\left(\beta_{1}+k\right) B_{2}^{2}}{32 \beta_{1}^{2} \varepsilon\left(4 \beta_{1}^{2}+8 \beta_{1} k+3 k^{2}\right)^{2}}$,

where

$$
\begin{aligned}
& A_{2}:=2\left[2 \beta_{1} \beta_{2}^{2} \ln \left(\frac{\bar{\mu}}{\bar{\mu}-\varepsilon}\right)+3 \beta_{2}^{2} k \ln \left(\frac{\bar{\mu}}{\bar{\mu}-\varepsilon}\right)+4 \alpha_{1} \beta_{1}^{2} \varepsilon+4 \alpha_{1} \beta_{1} \varepsilon k+2 \alpha_{2} \beta_{1} \varepsilon k\right], \quad \text { and } \\
& B_{2}:=4 \beta_{1} \beta_{2}^{2} \ln \left(\frac{\bar{\mu}}{\bar{\mu}-\varepsilon}\right)+4 \beta_{2}^{2} k \ln \left(\frac{\bar{\mu}}{\bar{\mu}-\varepsilon}\right)+\beta_{2}^{2} k \ln \left(\frac{\bar{\mu}+\varepsilon}{\bar{\mu}-\varepsilon}\right)+8 \alpha_{1} \beta_{1}^{2} \varepsilon+8 \alpha_{1} \beta_{1} \varepsilon k+4 \alpha_{2} \beta_{1} \varepsilon k
\end{aligned}
$$

Since $\left(A_{2}-B_{2}\right)=\beta_{2}^{2} k \ln \left(\frac{\bar{\mu}^{2}}{\bar{\mu}^{2}-\varepsilon^{2}}\right) \geq 0, A_{2}$ is larger than $B_{2}$. Thus, $\pi_{M 1}^{S h} \geq \pi_{M 1}^{P h}$.

For the second part, let $\pi_{M 1}^{S l}:=\frac{\left(\beta_{1}+k\right) C_{2}^{2}}{32 \varepsilon\left(4 \beta_{1}^{2}+8 \beta_{1} k+3 k^{2}\right)^{2}}$ and $\pi_{M 1}^{P l}:=\frac{\left(\beta_{1}+k\right) D_{2}^{2}}{32 \beta_{1}^{2} \varepsilon\left(4 \beta_{1}^{2}+8 \beta_{1} k+3 k^{2}\right)^{2}}$, where

$$
\begin{aligned}
& C_{2}:=2\left[2 \beta_{1} \beta_{2}^{2} \ln \left(\frac{\bar{\mu}+\varepsilon}{\bar{\mu}}\right)+3 \beta_{2}^{2} k \ln \left(\frac{\bar{\mu}+\varepsilon}{\bar{\mu}}\right)+4 \alpha_{1} \beta_{1}^{2} \varepsilon+4 \alpha_{1} \beta_{1} \varepsilon k+2 \alpha_{2} \beta_{1} \varepsilon k\right], \quad \text { and } \\
& D_{2}:=4 \beta_{1} \beta_{2}^{2} \ln \left(\frac{\bar{\mu}+\varepsilon}{\bar{\mu}}\right)+4 \beta_{2}^{2} k \ln \left(\frac{\bar{\mu}+\varepsilon}{\bar{\mu}}\right)+\beta_{2}^{2} k \ln \left(\frac{\bar{\mu}+\varepsilon}{\bar{\mu}-\varepsilon}\right)+8 \alpha_{1} \beta_{1}^{2} \varepsilon+8 \alpha_{1} \beta_{1} \varepsilon k+4 \alpha_{2} \beta_{1} \varepsilon k .
\end{aligned}
$$

Here $\left(D_{2}-C_{2}\right)=\beta_{2}^{2} k \ln \left(\frac{\bar{\mu}^{2}}{\bar{\mu}^{2}-\varepsilon^{2}}\right) \geq 0$ implying that $D_{2}$ is larger than $C_{2}$. Therefore, $\pi_{M 1}^{P l} \geq \pi_{M 1}^{S l}$. 


\section{A-9. Proof of Proposition 3}

Here also, substituting the optimal prices in profit expressions, we can derive that

$$
\begin{aligned}
& \pi_{M 2}^{N}=\frac{\left(\beta_{1}+k\right)\left[2 \beta_{1} \beta_{2}^{2} \ln \left(\frac{\bar{\mu}+\varepsilon}{\bar{\mu}-\varepsilon}\right)+3 \beta_{2}^{2} k \ln \left(\frac{\bar{\mu}+\varepsilon}{\bar{\mu}-\varepsilon}\right)+8 \alpha_{2} \beta_{1}^{2} \varepsilon+4 \alpha_{1} \beta_{1} \varepsilon k+8 \alpha_{2} \beta_{1} \varepsilon k\right]^{2}}{16 \beta_{1}^{2} \varepsilon\left(4 \beta_{1}^{2}+8 \beta_{1} k+3 k^{2}\right)^{2}}, \\
& \pi_{M 2}^{S h}=\frac{\left(\beta_{1}+k\right)\left[2 \beta_{1} \beta_{2}^{2} \ln \left(\frac{\bar{\mu}}{\bar{\mu}-\varepsilon}\right)+3 \beta_{2}^{2} k \ln \left(\frac{\bar{\mu}}{\bar{\mu}-\varepsilon}\right)+4 \alpha_{2} \beta_{1}^{2} \varepsilon+2 \alpha_{1} \beta_{1} \varepsilon k+4 \alpha_{2} \beta_{1} \varepsilon k\right]^{2}}{8 \beta_{1}^{2} \varepsilon\left(4 \beta_{1}^{2}+8 \beta_{1} k+3 k^{2}\right)^{2}}, \\
& \pi_{M 2}^{S l}=\frac{\left(\beta_{1}+k\right)\left[2 \beta_{1} \beta_{2}^{2} \ln \left(\frac{\bar{\mu}+\varepsilon}{\bar{\mu}}\right)+3 \beta_{2}^{2} k \ln \left(\frac{\bar{\mu}+\varepsilon}{\bar{\mu}}\right)+4 \alpha_{2} \beta_{1}^{2} \varepsilon+2 \alpha_{1} \beta_{1} \varepsilon k+4 \alpha_{2} \beta_{1} \varepsilon k\right]^{2}}{8 \beta_{1}^{2} \varepsilon\left(4 \beta_{1}^{2}+8 \beta_{1} k+3 k^{2}\right)^{2}}, \\
& \pi_{M 2}^{P h}=\frac{\left(\beta_{1}+k\right)\left[\beta_{1} \beta_{2}^{2} \ln \left(\frac{\bar{\mu}+\varepsilon}{\bar{\mu}-\varepsilon}\right)+\beta_{2}^{2} k \ln \left(\frac{\bar{\mu}+\varepsilon}{\bar{\mu}-\varepsilon}\right)+\beta_{2}^{2} k \ln \left(\frac{\bar{\mu}}{\bar{\mu}-\varepsilon}\right)+4 \alpha_{2} \beta_{1}^{2} \varepsilon+2 \alpha_{1} \beta_{1} \varepsilon k+4 \alpha_{2} \beta_{1} \varepsilon k\right]^{2}}{4 \beta_{1}^{2} \varepsilon\left(4 \beta_{1}^{2}+8 \beta_{1} k+3 k^{2}\right)^{2}}, \\
& \pi_{M 2}^{P h}=\frac{\left(\beta_{1}+k\right)\left[\beta_{1} \beta_{2}^{2} \ln \left(\frac{\bar{\mu}+\varepsilon}{\bar{\mu}-\varepsilon}\right)+\beta_{2}^{2} k \ln \left(\frac{\bar{\mu}+\varepsilon}{\bar{\mu}}\right)+\beta_{2}^{2} k \ln \left(\frac{\bar{\mu}+\varepsilon}{\bar{\mu}-\varepsilon}\right)+4 \alpha_{2}^{2}+8 \beta_{1} k+3 k_{1}^{2}\right)^{2}}{\left.42 \alpha_{1} \beta_{1} \varepsilon k+4 \alpha_{2} \beta_{1} \varepsilon k\right]^{2}} .
\end{aligned}
$$

First, let $\pi_{M 2}^{N}:=\frac{\left(\beta_{1}+k\right) A_{3}^{2}}{16 \beta_{1}^{2} \varepsilon\left(4 \beta_{1}^{2}+8 \beta_{1} k+3 k^{2}\right)^{2}}$ and $\pi_{M 2}^{S h}:=\frac{\left(\beta_{1}+k\right) B_{3}^{2}}{16 \beta_{1}^{2} \varepsilon\left(4 \beta_{1}^{2}+8 \beta_{1} k+3 k^{2}\right)^{2}}$,

where

$$
\begin{aligned}
& A_{3}:=2 \beta_{1} \beta_{2}^{2} \ln \left(\frac{\bar{\mu}+\varepsilon}{\bar{\mu}-\varepsilon}\right)+3 \beta_{2}^{2} k \ln \left(\frac{\bar{\mu}+\varepsilon}{\bar{\mu}-\varepsilon}\right)+8 \alpha_{2} \beta_{1}^{2} \varepsilon+4 \alpha_{1} \beta_{1} \varepsilon k+8 \alpha_{2} \beta_{1} \varepsilon k, \text { and } \\
& B_{3}:=\sqrt{2}\left[2 \beta_{1} \beta_{2}^{2} \ln \left(\frac{\bar{\mu}}{\bar{\mu}-\varepsilon}\right)+3 \beta_{2}^{2} k \ln \left(\frac{\bar{\mu}}{\bar{\mu}-\varepsilon}\right)+4 \alpha_{2} \beta_{1}^{2} \varepsilon+2 \alpha_{1} \beta_{1} \varepsilon k+4 \alpha_{2} \beta_{1} \varepsilon k\right] .
\end{aligned}
$$

Here also it is easy to see that for sufficiently large $\alpha_{i}(i=1,2), A_{3} \geq B_{3}$, and therefore, $\pi_{M 2}^{N} \geq \pi_{M 2}^{S h}$. A similar argument yields $\pi_{M 2}^{N} \geq \pi_{M 2}^{S l}$. Thus, we have $\pi_{M 2}^{N} \geq \pi_{M 2}^{S}$.

The proof $\pi_{M 2}^{P} \geq \pi_{M 2}^{S}$ is similar and hence, we have omitted it. 


\section{A-10. Proof of Proposition 4}

It can be shown that

$$
\begin{aligned}
& \pi_{M 2}^{N}=\frac{\left(\beta_{1}+k\right) A_{4}^{2}}{16 \beta_{1}^{2} \varepsilon\left(4 \beta_{1}^{2}+8 \beta_{1} k+3 k^{2}\right)^{2}} ; \quad \pi_{M 2}^{P h}=\frac{\left(\beta_{1}+k\right) C_{4}^{2}}{16 \beta_{1}^{2} \varepsilon\left(4 \beta_{1}^{2}+8 \beta_{1} k+3 k^{2}\right)^{2}} ; \\
& \pi_{M 2}^{P l}=\frac{\left(\beta_{1}+k\right) D_{4}^{2}}{16 \beta_{1}^{2} \varepsilon\left(4 \beta_{1}^{2}+8 \beta_{1} k+3 k^{2}\right)^{2}},
\end{aligned}
$$

where

$$
\begin{aligned}
& A_{4}:=2 \beta_{1} \beta_{2}^{2} \ln \left(\frac{\bar{\mu}+\varepsilon}{\bar{\mu}-\varepsilon}\right)+3 \beta_{2}^{2} k \ln \left(\frac{\bar{\mu}+\varepsilon}{\bar{\mu}-\varepsilon}\right)+8 \alpha_{2} \beta_{1}^{2} \varepsilon+4 \alpha_{1} \beta_{1} \varepsilon k+8 \alpha_{2} \beta_{1} \varepsilon k, \\
& C_{4}:=2\left[\beta_{1} \beta_{2}^{2} \ln \left(\frac{\bar{\mu}+\varepsilon}{\bar{\mu}-\varepsilon}\right)+\beta_{2}^{2} k \ln \left(\frac{\bar{\mu}+\varepsilon}{\bar{\mu}-\varepsilon}\right)+\beta_{2}^{2} k \ln \left(\frac{\bar{\mu}}{\bar{\mu}-\varepsilon}\right)+4 \alpha_{2} \beta_{1}^{2} \varepsilon+2 \alpha_{1} \beta_{1} \varepsilon k+4 \alpha_{2} \beta_{1} \varepsilon k\right], \quad \text { and } \\
& D_{4}:=2\left[\beta_{1} \beta_{2}^{2} \ln \left(\frac{\bar{\mu}+\varepsilon}{\bar{\mu}-\varepsilon}\right)+\beta_{2}^{2} k \ln \left(\frac{\bar{\mu}+\varepsilon}{\bar{\mu}}\right)+\beta_{2}^{2} k \ln \left(\frac{\bar{\mu}+\varepsilon}{\bar{\mu}-\varepsilon}\right)+4 \alpha_{2} \beta_{1}^{2} \varepsilon+2 \alpha_{1} \beta_{1} \varepsilon k+4 \alpha_{2} \beta_{1} \varepsilon k\right] .
\end{aligned}
$$

As in the proofs of the previous propositions, it is easy to see that $C_{4} \geq A_{4}$ and $A_{4} \geq D_{4}$. Thus, we have the desired result, i.e., $\pi_{M 2}^{P h} \geq \pi_{M 2}^{N}$ and $\pi_{M 2}^{N} \geq \pi_{M 2}^{P l}$.

\section{A-11. Proof of Proposition 5}

It can be shown that

$$
\begin{gathered}
\pi_{R}^{N}=\frac{\left(4 \beta^{2} \varepsilon+2 \beta \varepsilon k-\beta^{2} \mu_{1} \ln \left(\frac{\bar{\mu}+\varepsilon}{\bar{\mu}-\varepsilon}\right)+4 \alpha \varepsilon k \mu_{1}+4 \alpha \beta \varepsilon \mu_{1}\right)^{2}}{32 \beta \varepsilon^{2} \mu_{1}^{2}(2 \beta+\mathrm{k})^{2}}, \\
\pi_{R}^{S \mathrm{~h}}=\frac{\left(2 \beta^{2} \varepsilon+\beta \varepsilon k-\beta^{2} \mu_{1} \ln \left(\frac{\bar{\mu}}{\bar{\mu}-\varepsilon}\right)+2 \alpha \varepsilon k \mu_{1}+2 \alpha \beta \varepsilon \mu_{1}\right)^{2}}{8 \beta \varepsilon^{2} \mu_{1}^{2}(2 \beta+\mathrm{k})^{2}}, \\
\pi_{R}^{S l}=\frac{\left(2 \beta^{2} \varepsilon+\beta \varepsilon k-\beta^{2} \mu_{1} \ln \left(\frac{\bar{\mu}+\varepsilon}{\bar{\mu}}\right)+2 \alpha \varepsilon k \mu_{1}+2 \alpha \beta \varepsilon \mu_{1}\right)^{2}}{8 \beta \varepsilon^{2} \mu_{1}^{2}(2 \beta+\mathrm{k})^{2}}, \\
\pi_{R}^{P h}=\frac{(\beta+2 k)^{2} A}{64 \beta \varepsilon^{2} \mu_{1}^{2}\left(4 \beta^{3}+16 \beta^{2} k+19 \beta k^{2}+6 k^{3}\right)^{2}}, \\
\pi_{R}^{P l}=\frac{(\beta+2 k)^{2} B}{64 \beta \varepsilon^{2} \mu_{1}^{2}\left(4 \beta^{3}+16 \beta^{2} k+19 \beta k^{2}+6 k^{3}\right)^{2}}
\end{gathered}
$$


where

Let $\pi_{R}^{N}=\frac{A_{5}^{2}}{32 \beta \varepsilon^{2} \mu_{1}^{2}(2 \beta+k)^{2}}$ and $\pi_{R}^{S l}=\frac{B_{5}^{2}}{8 \beta \varepsilon^{2} \mu_{1}^{2}(2 \beta+k)^{2}}$,

where

$\mathrm{A}=128 \beta^{6} \varepsilon^{2}+512 \beta^{5} \varepsilon^{2} \mathrm{k}+4 \beta^{6} \varepsilon^{2}\left(\ln \left(\frac{\bar{\mu}+\varepsilon}{\bar{\mu}-\varepsilon}\right)\right)^{2}+16 \beta^{6} \varepsilon^{2}\left(\ln \left(\frac{\bar{\mu}}{\bar{\mu}-\varepsilon}\right)\right)^{2}+72 \beta^{2} \varepsilon^{2} \mathrm{k}^{4}+384 \beta^{3} \varepsilon^{2} \mathbf{k}^{3}+704 \beta^{4} \varepsilon^{2} \mathbf{k}^{2}$

$+\beta^{3} k^{3} \mu_{1}^{2}\left(\ln \left(\frac{\bar{\mu}+\varepsilon}{\bar{\mu}-\varepsilon}\right)\right)^{2}+9 \beta^{4} k^{2} \mu_{1}^{2}\left(\ln \left(\frac{\bar{\mu}+\varepsilon}{\bar{\mu}-\varepsilon}\right)\right)^{2}-64 \beta^{6} \varepsilon \mu_{1} \ln \frac{\bar{\mu}}{\bar{\mu}-\varepsilon}+4 \beta^{3} k^{3} \mu_{1}^{2}\left(\ln \left(\frac{\bar{\mu}}{\bar{\mu}-\varepsilon}\right)\right)^{2}+36 \beta^{4} k^{2} \mu_{1}^{2}\left(\ln \left(\frac{\bar{\mu}}{\bar{\mu}-\varepsilon}\right)\right)^{2}$

$+128 \alpha^{2} \beta^{4} \varepsilon^{2} \mu_{1}^{2}+288 \alpha^{2} \varepsilon^{2} k^{4} \mu_{1}^{2}+256 \alpha \beta^{5} \varepsilon^{2} \mu_{1}+12 \beta^{5} \alpha \mu_{1}\left(\ln \left(\frac{\bar{\mu}+\varepsilon}{\bar{\mu}-\varepsilon}\right)\right)^{2}-32 \beta^{6} \varepsilon \mu_{1} \ln \frac{\bar{\mu}+\varepsilon}{\bar{\mu}-\varepsilon}+48 \beta^{5} k \mu_{1}^{2}\left(\ln \left(\frac{\bar{\mu}}{\bar{\mu}-\varepsilon}\right)\right)^{2}$

$+288 \alpha \beta \varepsilon^{2} k^{4} \mu_{1}+1152 \alpha \beta^{4} \varepsilon^{2} k \mu_{1}+1184 \alpha^{2} \beta^{4} \varepsilon^{2} k^{2} \mu_{1}^{2}-112 \beta^{5} \varepsilon k \mu_{1} \ln \frac{\bar{\mu}+\varepsilon}{\bar{\mu}-\varepsilon}+1248 \alpha \beta^{2} \varepsilon^{2} k^{3} \mu_{1}+1856 \alpha \beta^{3} \varepsilon^{2} k^{2} \mu_{1}$

$-224 \beta^{5} \varepsilon k \mu_{1} \ln \frac{\bar{\mu}}{\bar{\mu}-\varepsilon}-32 \alpha \beta^{5} \varepsilon \mu_{1}^{2} \ln \frac{\bar{\mu}+\varepsilon}{\bar{\mu}-\varepsilon}-36 \beta^{3} \varepsilon k^{3} \mu_{1} \ln \frac{\bar{\mu}+\varepsilon}{\bar{\mu}-\varepsilon}-120 \beta^{4} \varepsilon k^{2} \mu_{1} \ln \frac{\bar{\mu}+\varepsilon}{\bar{\mu}-\varepsilon}+960 \alpha^{2} \beta \varepsilon^{2} k^{3} \mu_{1}^{2}$

$+640 \alpha^{2} \beta^{3} \varepsilon^{2} k \mu_{1}^{2}-64 \alpha \beta^{5} \varepsilon \mu_{1}^{2} \ln \frac{\bar{\mu}}{\bar{\mu}-\varepsilon}-4 \beta^{3} k^{3} \mu_{1}^{2} \ln \frac{\bar{\mu}}{\bar{\mu}-\varepsilon} \ln \frac{\bar{\mu}+\varepsilon}{\bar{\mu}-\varepsilon}-72 \beta^{3} \varepsilon k^{3} \mu_{1} \ln \frac{\bar{\mu}}{\bar{\mu}-\varepsilon}-240 \beta^{4} \varepsilon k^{2} \mu_{1} \ln \frac{\bar{\mu}}{\bar{\mu}-\varepsilon}$

$-128 \alpha \beta^{4} \varepsilon k \mu_{1}^{2} \ln \frac{\bar{\mu}+\varepsilon}{\bar{\mu}-\varepsilon}-256 \alpha \beta^{4} \varepsilon k \mu_{1}^{2} \ln \frac{\bar{\mu}}{\bar{\mu}-\varepsilon}-168 \alpha \beta^{3} \varepsilon k^{2} \mu_{1}^{2} \ln \frac{\bar{\mu}+\varepsilon}{\bar{\mu}-\varepsilon}-144 \alpha \beta^{2} \varepsilon k^{3} \mu_{1}^{2} \ln \frac{\bar{\mu}}{\bar{\mu}-\varepsilon}$

$-336 \alpha \beta^{3} \varepsilon k^{2} \mu_{1}^{2} \ln \frac{\bar{\mu}}{\bar{\mu}-\varepsilon}$,

$\mathrm{B}=128 \beta^{6} \varepsilon^{2}+512 \beta^{5} \varepsilon^{2} \mathrm{k}+4 \beta^{6} \mu_{1}^{2}\left(\ln \left(\frac{\bar{\mu}+\varepsilon}{\bar{\mu}-\varepsilon}\right)\right)^{2}+16 \beta^{6} \mu_{1}^{2}\left(\ln \left(\frac{\bar{\mu}}{\bar{\mu}-\varepsilon}\right)\right)^{2}+72 \beta^{2} \varepsilon^{2} \mathrm{k}^{4}+384 \beta^{3} \varepsilon^{2} \mathrm{k}^{3}+704 \beta^{4} \varepsilon^{2} \mathrm{k}^{2}$

$+\beta^{3} k^{3} \mu_{1}^{2}\left(\ln \left(\frac{\bar{\mu}+\varepsilon}{\bar{\mu}-\varepsilon}\right)\right)^{2}+9 \beta^{4} k^{2} \mu_{1}^{2}\left(\ln \left(\frac{\bar{\mu}+\varepsilon}{\bar{\mu}-\varepsilon}\right)\right)^{2}-64 \beta^{6} \varepsilon \mu_{1} \ln \frac{\bar{\mu}}{\bar{\mu}-\varepsilon}+16 \beta^{3} k^{3} \mu_{1}^{2}\left(\ln \left(\frac{\bar{\mu}}{\bar{\mu}-\varepsilon}\right)\right)^{2}+48 \beta^{4} k^{2} \mu_{1}^{2}\left(\ln \left(\frac{\bar{\mu}}{\bar{\mu}-\varepsilon}\right)\right)^{2}$

$+128 \alpha^{2} \beta^{4} \varepsilon^{2} \mu_{1}^{2}+288 \alpha^{2} \varepsilon^{2} k^{4} \mu_{1}^{2}+256 \alpha \beta^{5} \varepsilon^{2} \mu_{1}+12 \beta^{5} \mathrm{k} \mu_{1}^{2}\left(\ln \left(\frac{\bar{\mu}+\varepsilon}{\bar{\mu}-\varepsilon}\right)\right)^{2}-32 \beta^{6} \varepsilon \mu_{1} \ln \frac{\bar{\mu}+\varepsilon}{\bar{\mu}-\varepsilon}+48 \beta^{5} k \mu_{1}^{2}\left(\ln \left(\frac{\bar{\mu}}{\bar{\mu}-\varepsilon}\right)\right)^{2}$

$+4 \beta^{3} k^{3} \mu_{1}^{2}\left(\ln \left(\frac{\bar{\mu}+\varepsilon}{\bar{\mu}}\right)\right)^{2}+4 \beta^{4} k^{2} \mu_{1}^{2}\left(\ln \left(\frac{\bar{\mu}+\varepsilon}{\bar{\mu}}\right)\right)^{2}+288 \alpha \beta \varepsilon^{2} k^{4} \mu_{1}+1152 \alpha \beta^{4} \varepsilon^{2} k \mu_{1}+8 \beta^{5} k \mu_{1}^{2} \ln \frac{\bar{\mu}+\varepsilon}{\bar{\mu}} \ln \frac{\bar{\mu}+\varepsilon}{\bar{\mu}-\varepsilon}$

$-32 \beta^{5} \varepsilon k \mu_{1} \ln \frac{\bar{\mu}+\varepsilon}{\bar{\mu}}+1184 \alpha^{2} \beta^{2} \varepsilon^{2} k^{2} \mu_{1}^{2}-112 \beta^{5} \varepsilon k \mu_{1} \ln \frac{\bar{\mu}+\varepsilon}{\bar{\mu}-\varepsilon}+1248 \alpha \beta^{2} \varepsilon^{2} k^{3} \mu_{1}+1856 \alpha \beta^{3} \varepsilon^{2} k^{2} \mu_{1}$

$-8 \beta^{5} k \mu_{1}^{2} \ln \frac{\bar{\mu}}{\bar{\mu}-\varepsilon} \ln \frac{\bar{\mu}+\varepsilon}{\bar{\mu}+\varepsilon}+4 \beta^{3} k^{3} \mu_{1}^{2} \ln \frac{\bar{\mu}+\varepsilon}{\bar{\mu}} \ln \frac{\bar{\mu}+\varepsilon}{\bar{\mu}+\varepsilon}+16 \beta^{4} k^{2} \mu_{1}^{2} \ln \frac{\bar{\mu}+\varepsilon}{\bar{\mu}} \ln \frac{\bar{\mu}+\varepsilon}{\bar{\mu}+\varepsilon}-192 \beta^{5} \varepsilon k \mu_{1} \ln \frac{\bar{\mu}}{\bar{\mu}-\varepsilon}$

$-24 \beta^{3} \varepsilon k^{3} \mu_{1} \ln \frac{\bar{\mu}+\varepsilon}{\bar{\mu}}-64 \beta^{4} \varepsilon k^{2} \mu_{1} \ln \frac{\bar{\mu}+\varepsilon}{\bar{\mu}}-32 \alpha \beta^{5} \varepsilon \mu_{1}^{2} \ln \frac{\bar{\mu}+\varepsilon}{\bar{\mu}-\varepsilon}-36 \beta^{3} \varepsilon k^{3} \mu_{1} \ln \frac{\bar{\mu}+\varepsilon}{\bar{\mu}-\varepsilon}-120 \beta^{4} \varepsilon k^{2} \mu_{1} \ln \frac{\bar{\mu}+\varepsilon}{\bar{\mu}-\varepsilon}$

$-16 \beta^{3} k^{3} \mu_{1}^{2} \ln \frac{\bar{\mu}+\varepsilon}{\bar{\mu}} \ln \frac{\bar{\mu}}{\bar{\mu}-\varepsilon}-16 \beta^{4} k^{2} \mu_{1}^{2} \ln \frac{\bar{\mu}+\varepsilon}{\bar{\mu}} \ln \frac{\bar{\mu}}{\bar{\mu}-\varepsilon}+960 \alpha^{2} \beta \varepsilon^{2} k^{3} \mu_{1}^{2}+640 \alpha^{2} \beta^{3} \varepsilon^{2} k \mu_{1}^{2}-64 \alpha \beta^{5} \varepsilon \mu_{1}^{2} \ln \frac{\bar{\mu}}{\bar{\mu}-\varepsilon}$

$-8 \beta^{3} k^{3} \mu_{1}^{2} \ln \frac{\bar{\mu}}{\bar{\mu}-\varepsilon} \ln \frac{\bar{\mu}+\varepsilon}{\bar{\mu}-\varepsilon}-16 \beta^{4} k^{2} \mu_{1}^{2} \ln \frac{\bar{\mu}+\varepsilon}{\bar{\mu}} \ln \frac{\bar{\mu}}{\bar{\mu}-\varepsilon}-48 \beta^{3} \varepsilon k^{3} \mu_{1} \ln \frac{\bar{\mu}}{\bar{\mu}-\varepsilon}-176 \beta^{4} \varepsilon k^{3} \mu_{1} \ln \frac{\bar{\mu}}{\bar{\mu}-\varepsilon}$

$-32 \alpha \beta^{4} \varepsilon k \mu_{1}^{2} \ln \frac{\bar{\mu}+\varepsilon}{\bar{\mu}}-128 \alpha \beta^{4} \varepsilon k \mu_{1}^{2} \ln \frac{\bar{\mu}+\varepsilon}{\bar{\mu}-\varepsilon}-224 \alpha \beta^{4} \varepsilon k \mu_{1}^{2} \ln \frac{\bar{\mu}}{\bar{\mu}-\varepsilon}-48 \alpha \beta^{2} \varepsilon k^{3} \mu_{1}^{2} \ln \frac{\bar{\mu}+\varepsilon}{\bar{\mu}}$

$-80 \alpha \beta^{3} \varepsilon k^{2} \mu_{1}^{2} \ln \frac{\bar{\mu}+\varepsilon}{\bar{\mu}}-72 \alpha \beta^{2} \varepsilon k^{3} \mu_{1}^{2} \ln \frac{\bar{\mu}+\varepsilon}{\bar{\mu}-\varepsilon}-168 \alpha \beta^{3} \varepsilon k^{2} \mu_{1}^{2} \ln \frac{\bar{\mu}+\varepsilon}{\bar{\mu}-\varepsilon}-96 \alpha \beta^{2} \varepsilon k^{3} \mu_{1}^{2} \ln \frac{\bar{\mu}}{\bar{\mu}-\varepsilon}$

$-256 \alpha \beta^{3} \varepsilon k^{2} \mu_{1}^{2} \ln \frac{\bar{\mu}}{\bar{\mu}-\varepsilon}$. 


$$
\begin{aligned}
& A_{5}=4 \beta^{2} \varepsilon+2 \beta \varepsilon \mathrm{k}-\beta^{2} \mu_{1} \ln \left(\frac{\bar{\mu}+\varepsilon}{\bar{\mu}-\varepsilon}\right)+4 \alpha \varepsilon k \mu_{1}+4 \alpha \beta \varepsilon \mu_{1}, \\
& B_{5}=2\left[2 \beta^{2} \varepsilon+\beta \varepsilon k-\beta^{2} \mu_{1} \ln \left(\frac{\bar{\mu}+\varepsilon}{\bar{\mu}}\right)+2 \alpha \varepsilon k \mu_{1}+2 \alpha \beta \varepsilon \mu_{1}\right] .
\end{aligned}
$$

Since $\left(B_{5}-A_{5}\right)=\beta^{2} \mu_{1} \ln \left(\frac{\bar{\mu}}{\bar{\mu}-\varepsilon}\right) \geq 0, B_{5} \geq A_{5}$. Thus, $\pi_{R}^{S l} \geq \pi_{R}^{N}$.

Next, note that $\pi_{R}^{N}-\pi_{R}^{P l}=\frac{\beta(\beta+2 k)^{2} C_{5}}{64 \varepsilon^{2} \mu_{1}\left(4 \beta^{3}+16 \beta^{2}+19 \beta^{2} k+6 k^{3}\right)^{2}}$,

where

$$
\begin{aligned}
C_{5}= & 64 \beta^{4} \varepsilon \ln \frac{\bar{\mu}}{\bar{\mu}-\varepsilon}-32 \beta^{4} \varepsilon \ln \frac{\bar{\mu}+\varepsilon}{\bar{\mu}-\varepsilon}+16 \beta^{4} \mu_{1}\left(\ln \left(\frac{\bar{\mu}}{\bar{\mu}-\varepsilon}\right)\right)^{2}+4 \beta^{4} \mu_{1}\left(\ln \left(\frac{\bar{\mu}+\varepsilon}{\bar{\mu}-\varepsilon}\right)\right)^{2}+48 \beta \varepsilon k^{3} \ln \frac{\bar{\mu}}{\bar{\mu}-\varepsilon} \\
& +192 \beta^{3} \varepsilon k \ln \frac{\bar{\mu}}{\bar{\mu}-\varepsilon}+64 \beta^{2} \varepsilon k^{2} \ln \frac{\bar{\mu}+\varepsilon}{\bar{\mu}}-4 \beta k^{3} \mu_{1}\left(\ln \frac{\bar{\mu}+\varepsilon}{\bar{\mu}}\right)^{2}-120 \beta^{2} \varepsilon k^{2} \ln \frac{\bar{\mu}+\varepsilon}{\bar{\mu}-\varepsilon}-\beta k^{3} \mu_{1}\left(\ln \frac{\bar{\mu}+\varepsilon}{\bar{\mu}-\varepsilon}\right)^{2} \\
& +12 \beta^{3} k \mu_{1}\left(\ln \frac{\bar{\mu}+\varepsilon}{\bar{\mu}-\varepsilon}\right)^{2}+176 \beta^{2} \varepsilon k^{2} \ln \frac{\bar{\mu}}{\bar{\mu}-\varepsilon}-16 \beta k^{3} \mu_{1}\left(\ln \frac{\bar{\mu}}{\bar{\mu}-\varepsilon}\right)^{2}-48 \beta^{3} k \mu_{1}\left(\ln \frac{\bar{\mu}}{\bar{\mu}-\varepsilon}\right)^{2}-4 \beta^{2} k^{2} \mu_{1}\left(\ln \frac{\bar{\mu}+\varepsilon}{\bar{\mu}}\right)^{2} \\
& +9 \beta^{2} k^{2} \mu_{1}\left(\ln \frac{\bar{\mu}+\varepsilon}{\bar{\mu}-\varepsilon}\right)^{2}+24 \beta \varepsilon k^{3} \ln \frac{\bar{\mu}+\varepsilon}{\bar{\mu}}+32 \beta^{3} \varepsilon k \ln \frac{\bar{\mu}+\varepsilon}{\bar{\mu}}-36 \beta \varepsilon k^{3} \ln \frac{\bar{\mu}+\varepsilon}{\bar{\mu}-\varepsilon}-112 \beta^{3} \varepsilon k \ln \frac{\bar{\mu}+\varepsilon}{\bar{\mu}-\varepsilon} \\
& -48 \beta^{2} k^{2} \mu_{1}\left(\ln \frac{\bar{\mu}}{\bar{\mu}-\varepsilon}\right)^{2}+16 \beta k^{3} \mu_{1} \ln \frac{\bar{\mu}}{\bar{\mu}-\varepsilon} \ln \frac{\bar{\mu}+\varepsilon}{\bar{\mu}}+8 \beta k^{3} \ln \frac{\bar{\mu}}{\bar{\mu}-\varepsilon} \ln \frac{\bar{\mu}+\varepsilon}{\bar{\mu}-\varepsilon}+8 \beta^{3} k \mu_{1} \ln \frac{\bar{\mu}}{\bar{\mu}-\varepsilon} \ln \frac{\bar{\mu}+\varepsilon}{\bar{\mu}-\varepsilon} \\
& -16 \beta^{2} k^{2} \mu_{1} \ln \frac{\bar{\mu}+\varepsilon}{\bar{\mu}} \ln \frac{\bar{\mu}+\varepsilon}{\bar{\mu}-\varepsilon}+48 \alpha \varepsilon k^{3} \mu_{1} \ln \frac{\bar{\mu}+\varepsilon}{\bar{\mu}}-32 \alpha \beta^{3} \varepsilon \mu_{1} \ln \frac{\bar{\mu}+\varepsilon}{\bar{\mu}-\varepsilon}-72 \alpha \varepsilon k^{3} \ln \frac{\bar{\mu}+\varepsilon}{\bar{\mu}-\varepsilon} \\
& +16 \beta^{2} k^{2} \mu_{1} \ln \frac{\bar{\mu}+\varepsilon}{\bar{\mu}} \ln \frac{\bar{\mu}}{\bar{\mu}-\varepsilon}+64 \alpha \beta^{3} \varepsilon \mu_{1} \ln \frac{\bar{\mu}}{\bar{\mu}-\varepsilon}+16 \beta^{2} k^{2} \mu_{1} \ln \frac{\bar{\mu}+\varepsilon}{\bar{\mu}} \ln \frac{\bar{\mu}}{\bar{\mu}-\varepsilon}+64 \alpha \beta^{3} \varepsilon \mu_{1} \ln \frac{\bar{\mu}}{\bar{\mu}-\varepsilon} \\
& +16 \beta^{2} k^{2} \mu_{1} \ln \frac{\bar{\mu}+\varepsilon}{\bar{\mu}-\varepsilon} \ln \frac{\bar{\mu}}{\bar{\mu}-\varepsilon}+96 \alpha k^{3} \mu_{1} \ln \frac{\bar{\mu}}{\bar{\mu}-\varepsilon}-4 \beta k^{3} \mu_{1} \ln \frac{\bar{\mu}+\varepsilon}{\bar{\mu}} \ln \frac{\bar{\mu}+\varepsilon}{\bar{\mu}-\varepsilon}-8 \beta^{3} k \mu_{1} \ln \frac{\bar{\mu}+\varepsilon}{\bar{\mu}} \ln \frac{\bar{\mu}+\varepsilon}{\bar{\mu}-\varepsilon} \\
& +256 \alpha \beta \varepsilon k^{2} \mu_{1} \ln \frac{\bar{\mu}}{\bar{\mu}-\varepsilon}+224 \alpha \beta^{2} \varepsilon k \mu_{1} \ln \frac{\bar{\mu}}{\bar{\mu}-\varepsilon}+80 \alpha \beta \varepsilon k^{2} \mu_{1} \ln \frac{\bar{\mu}+\varepsilon}{\bar{\mu}}+32 \alpha \beta^{2} \varepsilon k \mu_{1} \ln \frac{\bar{\mu}+\varepsilon}{\bar{\mu}} \\
& -168 \alpha \beta \varepsilon k^{2} \mu_{1} \ln \frac{\bar{\mu}+\varepsilon}{\bar{\mu}-\varepsilon}-128 \alpha \beta^{2} \varepsilon k \mu_{1} \ln \frac{\bar{\mu}+\varepsilon}{\bar{\mu}-\varepsilon} .
\end{aligned}
$$

Again, for sufficiently large values of $\alpha$, it can be shown that $C_{5} \geq 0$, and thus, we have $\pi_{R}^{N} \geq \pi_{R}^{P l}$.

\section{A-12. Proof of Proposition 6}

This proof is similar to that of Proposition 5.

A-13. Proof of $\left(\pi_{M 1}^{N}-\pi_{M 1}^{P h}\right)+\left(\pi_{R}^{N}-\pi_{R}^{P h}\right)>\left(\pi_{M 2}^{P h}-\pi_{M 2}^{N}\right)$

To prove this result, we show that $\left(\pi_{M 2}^{P h}-\pi_{M 2}^{N}\right)-\left(\pi_{M 1}^{N}-\pi_{M 1}^{P h}\right)-\left(\pi_{R}^{N}-\pi_{R}^{P h}\right)<0$. Using some basic algebra, we obtain

$$
\begin{aligned}
& \left(\pi_{M 2}^{P h}-\pi_{M 2}^{N}\right)-\left(\pi_{M 1}^{N}-\pi_{M 1}^{P h}\right)-\left(\pi_{R}^{N}-\pi_{R}^{P h}\right)=\frac{(\beta+2 k)^{2} A_{6}}{64 \varepsilon^{2} \mu_{1}\left(4 \beta^{3}+16 \beta^{2} k+19 \beta k^{2}+6 k^{3}\right)^{2}}, \\
& \text { where }
\end{aligned}
$$




$$
\begin{aligned}
& A_{6}=16 \beta^{5} \mu_{1}\left(\ln \frac{\bar{\mu}}{\bar{\mu}-\varepsilon}\right)^{2}+32 \beta^{5} \varepsilon \ln \frac{\bar{\mu}+\varepsilon}{\bar{\mu}-\varepsilon}-64 \beta^{5} \varepsilon \ln \frac{\bar{\mu}}{\bar{\mu}-\varepsilon}-4 \beta^{5} \mu_{1}\left(\ln \left(\frac{\bar{\mu}+\varepsilon}{\bar{\mu}-\varepsilon}\right)\right)^{2}-256 \alpha^{2} \beta^{3} \varepsilon^{3} \mu_{1}-576 \alpha^{2} \varepsilon^{3} k^{3} \mu_{1} \\
& -224 \beta^{4} \varepsilon k \ln \frac{\bar{\mu}}{\bar{\mu}-\varepsilon}+256 \beta^{4} \varepsilon \mu_{1} \ln \frac{\bar{\mu}}{\bar{\mu}-\varepsilon}+36 \beta^{2} \varepsilon k^{3} \ln \frac{\bar{\mu}+\varepsilon}{\bar{\mu}-\varepsilon}+120 \beta^{3} \varepsilon k^{2} \ln \frac{\bar{\mu}+\varepsilon}{\bar{\mu}-\varepsilon}-16 \beta^{5} \varepsilon \mu_{1}\left(\ln \frac{\bar{\mu}+\varepsilon}{\bar{\mu}-\varepsilon}\right)^{2}-12 \beta^{4} k \mu_{1}\left(\ln \frac{\bar{\mu}+\varepsilon}{\bar{\mu}-\varepsilon}\right)^{2} \\
& -72 \beta^{2} \varepsilon k^{3} \ln \frac{\bar{\mu}}{\bar{\mu}-\varepsilon}-240 \beta^{3} \varepsilon k^{2} \ln \frac{\bar{\mu}}{\bar{\mu}-\varepsilon}+48 \beta^{4} k \mu_{1}\left(\ln \frac{\bar{\mu}}{\bar{\mu}-\varepsilon}\right)^{2}+512 \alpha \beta^{3} \varepsilon^{3} \mu_{1}+\beta^{2} k^{3} \mu_{1}\left(\ln \frac{\bar{\mu}+\varepsilon}{\bar{\mu}-\varepsilon}\right)^{2}-9 \beta^{3} k^{2} \mu_{1}\left(\ln \frac{\bar{\mu}+\varepsilon}{\bar{\mu}-\varepsilon}\right)^{2} \\
& +576 \alpha \varepsilon^{2} k^{3} \mu_{1}+112 \beta^{4} \varepsilon k \ln \frac{\bar{\mu}+\varepsilon}{\bar{\mu}-\varepsilon}+4 \beta^{2} k^{3} \mu_{1}\left(\ln \frac{\bar{\mu}}{\bar{\mu}-\varepsilon}\right)^{2}+36 \beta^{3} k^{2} \mu_{1}\left(\ln \frac{\bar{\mu}}{\bar{\mu}-\varepsilon}\right)^{2}-56 \beta^{2} k^{3} \mu_{1}\left(\ln \frac{\bar{\mu}+\varepsilon}{\bar{\mu}-\varepsilon}\right)^{2} \\
& +1920 \alpha \beta \varepsilon^{2} k^{2} \mu_{1}+1792 \alpha \beta^{2} \varepsilon^{2} k \mu_{1}+32 \alpha \beta^{4} \varepsilon \mu_{1} \ln \frac{\bar{\mu}+\varepsilon}{\bar{\mu}-\varepsilon}+16 \beta^{2} \varepsilon k^{3} \mu_{1}\left(\ln \frac{\bar{\mu}}{\bar{\mu}-\varepsilon}\right)^{2}+16 \beta^{3} \varepsilon k^{2} \mu_{1}\left(\ln \frac{\bar{\mu}}{\bar{\mu}-\varepsilon}\right) \\
& -1344 \alpha^{2} \beta \varepsilon^{2} k^{2} \mu_{1}-1024 \alpha^{2} \beta^{2} \varepsilon^{3} k \mu_{1}-64 \alpha \beta^{4} \varepsilon \mu_{1} \ln \frac{\bar{\mu}}{\bar{\mu}-\varepsilon}-4 \beta^{2} k^{3} \mu_{1} \ln \frac{\bar{\mu}}{\bar{\mu}-\varepsilon} \ln \frac{\bar{\mu}+\varepsilon}{\bar{\mu}-\varepsilon}+288 \beta \varepsilon k^{3} \mu_{1} \ln \frac{\bar{\mu}}{\bar{\mu}-\varepsilon} \\
& +896 \beta^{3} \varepsilon k \mu_{1} \ln \frac{\bar{\mu}}{\bar{\mu}-\varepsilon}-128 \alpha \beta^{4} \varepsilon^{2} \mu_{1} \ln \frac{\bar{\mu}+\varepsilon}{\bar{\mu}-\varepsilon}-80 \beta^{4} \varepsilon k \mu_{1}\left(\ln \frac{\bar{\mu}+\varepsilon}{\bar{\mu}-\varepsilon}\right)^{2}+960 \beta^{2} \varepsilon k^{2} \mu_{1} \ln \frac{\bar{\mu}}{\bar{\mu}-\varepsilon}-144 \alpha \beta k^{3} \mu_{1} \ln \frac{\bar{\mu}}{\bar{\mu}-\varepsilon} \\
& -256 \alpha \beta^{3} \varepsilon k \mu_{1} \ln \frac{\bar{\mu}}{\bar{\mu}-\varepsilon}+168 \alpha \beta^{2} \varepsilon k^{2} \mu_{1} \ln \frac{\bar{\mu}+\varepsilon}{\bar{\mu}-\varepsilon}-384 \alpha \beta \varepsilon^{2} k^{3} \mu_{1} \ln \frac{\bar{\mu}+\varepsilon}{\bar{\mu}-\varepsilon}-576 \alpha \beta^{3} \varepsilon^{2} k \mu_{1} \ln \frac{\bar{\mu}+\varepsilon}{\bar{\mu}-\varepsilon}-336 \alpha \beta^{2} \varepsilon k^{2} \mu_{1} \ln \frac{\bar{\mu}}{\bar{\mu}-\varepsilon} \\
& +192 \alpha \beta \varepsilon^{2} k^{3} \mu_{1} \ln \frac{\bar{\mu}}{\bar{\mu}-\varepsilon}+128 \alpha \beta^{3} \varepsilon^{2} k \mu_{1} \ln \frac{\bar{\mu}}{\bar{\mu}-\varepsilon}-832 \alpha \beta^{2} \varepsilon^{2} k^{2} \mu_{1} \ln \frac{\bar{\mu}+\varepsilon}{\bar{\mu}-\varepsilon}+32 \beta^{4} \varepsilon k \mu_{1} \ln \frac{\bar{\mu}}{\bar{\mu}-\varepsilon} \ln \frac{\bar{\mu}+\varepsilon}{\bar{\mu}-\varepsilon} \\
& +320 \alpha \beta^{2} \varepsilon^{2} k^{2} \mu_{1} \ln \frac{\bar{\mu}}{\bar{\mu}-\varepsilon}+72 \alpha \beta \varepsilon k^{3} \mu_{1} \ln \frac{\bar{\mu}+\varepsilon}{\bar{\mu}-\varepsilon}+128 \alpha \beta^{3} \varepsilon k \mu_{1} \ln \frac{\bar{\mu}+\varepsilon}{\bar{\mu}-\varepsilon}+32 \beta^{2} \varepsilon k^{3} \mu_{1} \ln \frac{\bar{\mu}}{\bar{\mu}-\varepsilon}+64 \beta^{3} \varepsilon k^{2} \mu_{1} \ln \frac{\bar{\mu}}{\bar{\mu}-\varepsilon} \ln \frac{\bar{\mu}+\varepsilon}{\bar{\mu}-\varepsilon}
\end{aligned}
$$

For sufficiently large $\alpha$, it can be shown that $A_{6}<0$. Hence, we have the desired result.

\section{A-14. Proof of Proposition 9}

For given $\mathrm{w}_{1}$ and $\mathrm{w}_{2}$, we know that $\pi_{M_{1}}^{R S C}=\mathrm{w}_{1} d_{1}, \pi_{M_{2}}^{R S C}=\mathrm{w}_{2} d_{2}$,

$$
\pi_{R}^{R S C}=\left(\mathrm{p}_{1}-w_{1}-c_{i(v)}\right) d_{1}+\left(\mathrm{p}_{2}-w_{2}-c_{i(v)}\right) d_{2} \text { and } \pi_{S C}^{R S C}=\pi_{R}^{R S C}+\pi_{M_{1}}^{R S C}+\pi_{M_{2}}^{R S C} \text {. }
$$

Thus, we have the following results:

$$
\begin{aligned}
& \frac{\partial \pi_{S C}^{R S C}}{\partial w_{S C}} \leq 0 \text {, we can get that } w_{1} \geq a+b w_{2} \text {, that is } \pi_{S C}^{R S C} \text { decreases in } w_{1} . \\
& \frac{\partial \pi_{S C}^{R C}}{\partial w_{2}} \leq 0 \text {, we can get that } w_{2} \geq a+b w_{1} \text {, that is } \pi_{S C}^{R S C} \text { decreases in } w_{2} .
\end{aligned}
$$

The Hessian matrix is:

$$
H=\left[\begin{array}{ll}
\frac{\partial_{\pi_{S C}^{2}}^{2}}{\pi_{S C}} & \partial_{\pi_{S C}^{2}}^{2} \\
\partial_{\mathrm{w}_{1}^{2}} & \frac{\pi_{w_{1} w_{2}}}{\partial^{2}} \\
\frac{\pi_{S C}^{R S C}}{\partial_{w_{2} w_{1}}} & \frac{\partial_{S C}^{2}}{\partial_{\mathrm{w}_{2}^{2}}}
\end{array}\right]=\left[\begin{array}{ll}
-\frac{\beta_{1}}{2}-\frac{k}{2} & \frac{k}{2} \\
\frac{k}{2} & -\frac{\beta_{1}}{2}-\frac{k}{2}
\end{array}\right]
$$

and

$$
|H|=\left(-\frac{\beta_{1}}{2}-\frac{\mathrm{k}}{2}\right)^{2}-\left(\frac{k}{2}\right)^{2}=\frac{\beta_{1}^{2}}{4}+\frac{\beta_{1} k}{2}>o
$$

Therefore, we can show that the Hessian matrix $\mathrm{H}$ is a diagonally dominant matrix, which guarantees joint concavity of the function $\pi_{S C}^{R S C}\left(w_{1}, w_{2}\right)$. 
We can get that

$$
\left\{\begin{array}{l}
\frac{a}{1-b} \leq w_{1} \leq w_{1}^{N *} \\
a+b w_{1} \leq w_{2} \leq \frac{w_{1}-a}{b}
\end{array}\right.
$$

where $a=\frac{\beta_{1}^{2} \mu_{1}^{2} v^{2}+2 \beta_{1} \beta_{2} \mu_{1} v-3 \beta_{2}^{2}}{2 \beta_{1} \mu_{1}\left(k+\beta_{1}\right)}$ and $b=\frac{k}{k+\beta_{1}}$.

In that case, the profit of the supply chain decreases in $w_{1}$ and $w_{2}$. And we prove that $\pi_{S C}^{R S C} \geq \pi_{S C}^{N}$, that is the revenue sharing contract realizes supply chain coordination under complete efficient cost sharing scenario. Then we distribute the profits according to a certain proportion, purposing to achieve the optimal profit for each participant.

\section{A-15. Proof of condition 2.}

This proof is similar to that of Proposition 9

\section{A-16. The retailer's profit as ' $k$ ' increases}

See Fig. 7.

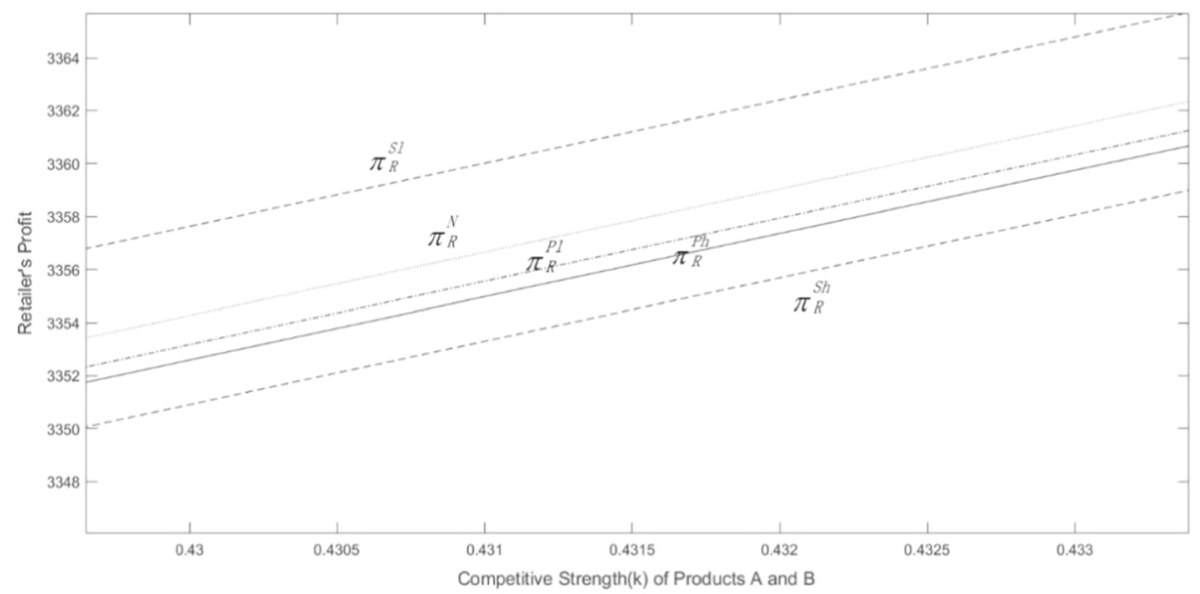

Fig. 7 The retailer's profit increases as the competition between products A and B characterized by $\mathrm{k}$ increases 
Acknowledgements We thank the guest editors for their precious time, and three anonymous reviewers for their valuable comments and suggestions. This work was supported by the National Natural Science Foundation (Grant number 71871091).

\section{References}

Armony, M., \& Haviv, M. (2003). Price and delay competition between two service providers. European Journal of Operational Research, 147(1), 32-50.

Briggs, F. (2015). Apple and John Lewis lead retail rankings For value added services, BookingBug study shows. Forbes. https://www.forbes.com/sites/fionabriggs/2015/12/07/apple-and-john-lewis -lead-retail-rankings-for-value-added-services-bookingbug-study-shows/?sh=73f754a3427d. Accessed date January 3, 2021.

Cai, K., He, Z., Lou, Y., \& He, S. (2019). Risk-aversion information in a supply chain with price and warranty competition. Annals of Operations Research, 287, 61-107.

Chen, W., \& Li, L. (2020). Incentive contracts for green building production with asymmetric information. International Journal of Production Research. https://doi.org/10.1080/00207543.2020.17270 47

Choi, S. C. (1991). Price Competition in a Channel Structure with a Common Retailer. Marketing Science, 10(4), 271-296.

Cohen, M. A., \& Whang, S. (1997). Competing in product and service: a product life-cycle model. Management Science, 43(4), 535-545.

Dai, Y., Zhou, S. X., \& Xu, Y. (2012). Competitive and collaborative quality and warranty management in supply chains. Production and Operations Management, 21(1), 129-144.

Dan, B., Zhang, S., \& Zhou, M. (2018). Strategies for warranty service in a dual-channel supply chain with value-added service competition. International Journal of Production Research, 56(17), 5677-5699.

Giri, B. C., \& Sarker, B. R. (2016). Coordinating a two-echelon supply chain under production disruption when retailers compete with price and service level. Operational Research, 16(1), 71-88.

Hartman, J. C., \& Laksana, K. (2009). Designing and pricing menus of extended warranty contracts. Naval Research Logistics, 56(3), 199-214.

Hu, W., Li, Y., \& Wang, W. (2017). Benefit and risk analysis of consignment contracts. Annals of Operations Research, 257, 641-659.

Kurata, H., \& Nam, S. H. (2010). After-sales service competition in a supply chain: Optimization of customer satisfaction level or profit or both? International Journal of Production Economics, 127(1), $136-146$.

Lau, A. H. L., Lau, H. S., \& Wang, J. C. (2007). Pricing and volume discounting for a dominant retailer with uncertain manufacturing cost information. European Journal of Operational Research, 183(2), 848-870.

Li, G., Huang, F. F., Cheng, T. C. E., Zheng, Q., \& Ji, P. (2014). Make-or-buy service capacity decision in a supply chain providing after-sales service. European Journal of Operational Research, 239(2), $377-388$.

Li, L. (2002). Information sharing in a supply chain with horizontal competition. Management Science, 48(9), 1196-1212.

Li, G., Li, L., \& Sun, J. (2019a). Pricing and service effort strategy in a dual-channel supply chain with showrooming effect. Transportation Research Part E: Logistics and Transportation Review, 126, 32-48.

Li, G., Li, L., Sethi, S. P., \& Guan, X. (2019b). Return strategy and pricing in a dual-channel supply chain. International Journal of Production Economics, 215, 153-164.

Li, G., Wu, H., \& Xiao, S. (2020). Financing strategies for a capital-constrained manufacturer in a dualchannel supply chain. International Transactions in Operational Research, 27, 2317-2339.

Li, L., \& Zhang, H. (2008). Confidentiality and information sharing in supply chain coordination. Management Science, 54(8), 1467-1481.

Li, G., Zheng, H., Sethi, S. P., \& Guan, X. (2020). Inducing downstream information sharing via manufacturer information acquisition and retailer subsidy. Decision Sciences, 51, 691-719.

Li, X. J., Chen, J., \& Ai, X. Z. (2019). Contract design in a cross-sales supply chain with demand information asymmetry. European Journal of Operational Research, 275(3), 939-956.

Lin, Y. T., \& Parlaktürk, A. (2012). Quick response under competition. Production and Operations Management, 21(3), 518-533. 
Liu, W., Wang, S., Zhu, D., Wang, D., \& Shen, X. (2018). Order allocation of logistics service supply chain with fairness concern and demand updating: model analysis and empirical examination. Annals of Operations Research, 268(1-2), 177-213.

Liu, Y., Li, J., Quan, B.-T., \& Yang, J.-B. (2019). Decision analysis and coordination of two-stage supply chain considering cost information asymmetry of corporate social responsibility. Journal of Cleaner Production, 228, 1073-1087.

Lu, J. C., Tsao, Y. C., \& Charoensiriwath, C. (2011). Competition under manufacturer service and retail price. Economic Modelling, 28(3), 1256-1264.

Lv, F., Xiao, L., Xu, M. H., \& Guan, X. (2019). Quantity-payment versus two-part tariff contracts in an assembly system with asymmetric cost information. Transportation Research Part E-Logistics and Transportation Review, 129, 60-80.

Ma, P., Shang, J., \& Wang, H. (2017). Enhancing corporate social responsibility: Contract design under information asymmetry. Omega, 67, 19-30.

Mehra, A., Kumar, S., \& Raju, J. S. (2017). Competitive strategies for brick-and-mortar stores to counter "showrooming." Management Science, 64(7), 3076-3090.

Mukhopadhyay, S. K., Zhu, X., \& Yue, X. (2008). Optimal contract design for mixed channels under information asymmetry. Production and Operations Management, 17(6), 641-650.

Sahin, F., \& Robinson, E. P. (2002). Flow coordination and information sharing in supply chains: Review, implications, and directions for future research. Decision Sciences, 33(4), 505-536.

Shang, W., Ha, A. Y., \& Tong, S. (2015). Information sharing in a supply chain with a common retailer. Management Science, 62(1), 245-263.

Shen, B., Choi, T. M., \& Minner, S. (2019). A review on supply chain contracting with information considerations: Information updating and information asymmetry. International Journal of Production Research, 57(15-16), 4898-4936.

Tsay, A. A., \& Agrawal, N. (2000). Channel dynamics under price and service competition. Manufacturing \& Service Operations Management, 2(4), 372-391.

Vosooghidizaji, M., Taghipour, A., \& Canel-Depitre, B. (2020). Supply chain coordination under information asymmetry: a review. International Journal of Production Research, 58(6), 1805-1834.

Xie, W., Jiang, Z., Zhao, Y., \& Shao, X. (2014). Contract design for cooperative product service system with information asymmetry. International Journal of Production Research, 52(6), 1658-1680.

Xie, W., Zhao, Y., Jiang, Z., \& Chow, P. (2016). Optimizing product service system by franchise fee contracts under information asymmetry. Annals of Operations Research, 240(2), 709-729.

Xing, W., Zhu, Q., \& Zhao, X. (2019). Supply contract design under price volatility and competition. International Journal of Production Research, 57, 1-16.

Yan, B., Chen, Z., Wang, X., \& Jin, Z. (2019). Influence of logistic service level on multichannel decision of a two-echelon supply chain. International Journal of Production Research, 58, 1-26.

Yan, B., Wang, T., Liu, Y. P., \& Liu, Y. (2016). Decision analysis of retailer-dominated dual-channel supply chain considering cost misreporting. International Journal of Production Economics, 178, 34-41.

Yao, D. Q., Yue, X., \& Liu, J. (2008). Vertical cost information sharing in a supply chain with value-adding retailers. Omega, 36(5), 838-851.

Zhang, J., \& Chen, J. (2013). Coordination of information sharing in a supply chain. International Journal of Production Economics, 143(1), 178-187.

Zhang, S., Dan, B., \& Zhou, M. (2019). After-sale service deployment and information sharing in a supply chain under demand uncertainty. European Journal of Operational Research., 279(2), 351-363.

Zhang, X., Han, X., Liu, X., Liu, R., \& Leng, J. (2015). The pricing of product and value-added service under information asymmetry: a product life cycle perspective. International Journal of Production Research, 53(1), 25-40.

Zhao, D., Zhang, X., Ren, T., \& Fu, H. (2019). Optimal Pricing strategies in a product and service supply chain with extended warranty service competition considering retailer fairness concern. Mathematical Problems in Engineering, 2019, 1-15.

Zhao, X., Xue, L., \& Zhang, F. (2014). Outsourcing competition and information sharing with asymmetrically informed suppliers. Production and Operations Management, 23(10), 1706-1718.

Zhou, M., Dan, B., Ma, S., \& Zhang, X. (2017). Supply chain coordination with information sharing: The informational advantage of GPOs. European Journal of Operational Research, 256(3), 785-802.

Publisher's Note Springer Nature remains neutral with regard to jurisdictional claims in published maps and institutional affiliations. 